\title{
Reversible inactivation of macaque frontal eye field
}

Received: 2 February 1996 / Accepted: 26 February 1997

\begin{abstract}
The macaque frontal eye field (FEF) is involved in the generation of saccadic eye movements and fixations. To better understand the role of the FEF, we reversibly inactivated a portion of it while a monkey made saccades and fixations in response to visual stimuli. Lidocaine was infused into a FEF and neural inactivation was monitored with a nearby microelectrode. We used two saccadic tasks. In the delay task, a target was presented and then extinguished, but the monkey was not allowed to make a saccade to its location until a cue to move was given. In the step task, the monkey was allowed to look at a target as soon as it appeared. During FEF inactivation, monkeys were severely impaired at making saccades to locations of extinguished contralateral targets in the delay task. They were similarly impaired at making saccades to locations of contralateral targets in the step task if the target was flashed for $\leq 100 \mathrm{~ms}$, such that it was gone before the saccade was initiated. Deficits included increases in saccadic latency, increases in saccadic error, and increases in the frequency of trials in which a saccade was not made. We varied the initial fixation location and found that the impairment specifically affected contraversive saccades rather than affecting all saccades made into head-centered contralateral space. Monkeys were impaired only slightly at making saccades to contralateral targets in the step task if the target duration was $1000 \mathrm{~ms}$, such that the target was present during the saccade: latency increased, but increases in saccadic error were mild and increases in the frequency of trials in which a saccade was not made were insignificant. During FEF inactivation there usually was a direct correla-
\end{abstract}

M. A. Sommer · E. J. Tehovnik

Department of Brain and Cognitive Sciences,

Massachusetts Institute of Technology,

Cambridge, MA 02139, USA

\section{A. Sommer (}

Laboratory of Sensorimotor Research, NEI,

National Institutes of Health, Building 49, Room 2A50,

9000 Rockville Pike, Bethesda, MD 20892-4435, USA

Tel.: +1 (301) 496-1141, Fax: +1 (301) 402-0511,

e-mail:mas@lsr.nei.nih.gov tion between the latency and the error of saccades made in response to contralateral targets. In the delay task, FEF inactivation increased the frequency of making premature saccades to ipsilateral targets. FEF inactivation had inconsistent and mild effects on saccadic peak velocity. FEF inactivation caused impairments in the ability to fixate lights steadily in contralateral space. FEF inactivation always caused an ipsiversive deviation of the eyes in darkness. In summary, our results suggest that the FEF plays major roles in (1) generating contraversive saccades to locations of extinguished or flashed targets, (2) maintaining contralateral fixations, and (3) suppressing inappropriate ipsiversive saccades.

Key words Saccadic eye movements · Fixations · Frontal eye field $\cdot$ Reversible inactivation .

Macaca mulatta

\section{Introduction}

A region of lateral frontal cortex in macaque, the frontal eye field (FEF), is involved in the generation of saccadic eye movements and fixations. Many neurons in the FEF discharge before and during contraversive saccades (Bruce and Goldberg 1985; Schall 1991). FEF presaccadic movement cells are tuned for direction and amplitude of contraversive saccades (Bruce and Goldberg 1985). Other FEF neurons fire during fixation (Bizzi 1968; Bizzi and Schiller 1970; Suzuki and Azuma 1977; Bruce and Goldberg 1985). FEF presaccadic movement neurons and fixation-related neurons project to subcortical oculomotor centers (Segraves and Goldberg 1987; Segraves 1992).

Electrical stimulation of FEF elicits contraversive saccades (Ferrier, 1875; Robinson and Fuchs 1969; Bruce et al. 1985). The direction and amplitude of saccades evoked from the FEF vary little with initial eye position (Robinson and Fuchs 1969; Goldberg and Bruce 1990; Schall, 1991; Russo and Bruce 1993), and prolonged stimulation causes the generation of repeated saccades of similar direction and amplitude (Robinson and Fuchs 
1969; Schiller 1977; Schiller et al. 1979a; Schall 1991) Therefore the FEF seems to command saccade generation with a "vector" code, directing the eyes to move a specific amount in a certain direction, rather than with a "place" code, directing the eyes to move to a particular location in the orbit.

Ablation of the FEF causes short-term effects that include a rise in threshold for brightness perception (Latto 1977), an impairment in the ability to react to briefly presented contralateral visual stimuli $(50 \mathrm{~ms}$ flashes, Latto and Cowey 1971a; 50-60 ms flashes, Schiller and Sandell 1983), a bias of fixation toward ipsilateral space (Latto and Cowey 1971b), and a decrease in the frequency of making contraversive saccades (Schiller et al. 1980). Most deficits of FEF ablation recover in a few weeks (Latto and Cowey 1971a,b; Latto 1977; Schiller et al. 1980), presumably because other brain areas take over the functions of the lesioned FEF. Effects of FEF ablation that have been reported to last beyond a month include the disability in making saccades to flashed targets (Schiller and Sandell 1983) and an impairment in learning to make contraversive saccades to locations of extinguished targets (Deng et al. 1986).

The purpose of the present study was to determine the oculomotor effects of reversible FEF inactivation. By temporarily silencing the FEF rather than ablating it, the chance that other brain areas will compensate for the loss of the FEF is minimized. This might permit a clearer understanding of the role of the FEF in the intact oculomotor system.

To inactivate the FEF we primarily used lidocaine, which binds to $\mathrm{Na}^{+}$channels and quickly inactivates neural tissue (Ritchie 1979; Ragsdale et al. 1994). While a monkey's FEF was inactivated, we assessed its ability to make saccades in response to visual stimuli and its ability to maintain fixation near visual stimuli. We varied initial eye position to examine whether effects caused by FEF inactivation were more consistent with vector coding or place coding. To ensure that effects were due to inactivation of FEF neurons, not fibers of passage, we used the GABA agonist muscimol as a control (GABA receptors are not found on axons). As a volume control we used saline.

The results of this study will be discussed in the context of the previous findings from FEF recording, stimulation, and ablation studies. Also, the results will be compared with previously reported results of inactivating an important subcortical oculomotor structure, the superior colliculus.

\section{Materials and methods}

\section{Monkeys}

Two monkeys (Macaca mulatta) were used ("L" and "I"). For surgery, a monkey was anesthetized with ketamine $(10 \mathrm{mg} / \mathrm{kg})$ followed by i.v. injection of pentobarbital $(30 \mathrm{mg} / \mathrm{kg})$. A search coil was implanted subconjunctivally (Robinson 1963; Judge et al. 1980 ) in the right eye. The skull was exposed, screws were put in normal to the skull, and acrylic cement was applied. A stainlesssteel post was set in the acrylic to restrain the head during testing. In a subsequent surgery a chamber was implanted to access the left FEF. Correct placement of the chamber was verified visually, since the arcuate and principal sulci could be identified through the dura. Monkeys received antibiotics and pain-killers (buprenorphine) postoperatively. They were allowed several days of recovery before testing. The monkeys were deprived of water overnight before testing and received apple juice reward during the experiments. The monkeys were provided for in accordance with the NIH Guide for the Care and Use of Laboratory Animals and the guidelines of the MIT Committee on Animal Care.

\section{Identification of the FEF with stimulation mapping}

The exact location of the FEF was identified with electrical stimulation. A Pt-Ir, glass-insulated microelectrode $(0.15-1.5 \mathrm{M} \Omega$ at $1 \mathrm{kHz}$ ) was introduced through the dura. Penetrations were made $1 \mathrm{~mm}$ apart in a grid pattern. Stimulation was performed at the first recorded unit(s) and then every $0.1 \mathrm{~mm}$. The stimulation parameters were those used by Bruce and Goldberg (1985): biphasic pulses, pulse duration of $0.25 \mathrm{~ms}$, frequency of $350 \mathrm{~Hz}$, and train duration of $70 \mathrm{~ms}$. We used a current of $150 \mu \mathrm{A}$ to determine the depth range for evoking saccades. Within this range we measured current thresholds. The core of the FEF has been defined as the periarcuate region within which saccades can be "evoked by electrical stimulation with currents $<50 \mu \mathrm{A}$ at some point in the electrode penetration" (Bruce and Goldberg 1985). We used seven such sites in each monkey for lidocaine, muscimol, or saline infusion (Fig. 1).

Saccades could be evoked only after the electrode tip was $\geq 1.5 \mathrm{~mm}$ below the first unit(s). Typically, saccades could be evoked until $\sim 8 \mathrm{~mm}$ below the first unit(s), indicating that the electrode tip followed the bank, or reached the buried bank, of the arcuate sulcus. Stimulation-evoked saccades were 3-30 deg in amplitude. Saccadic amplitude usually decreased with depth of penetration. Nearly all contraversive directions could be elicited in a single penetration. "Staircase" saccades were evoked by prolonged stimulation trains. In the arcuate region, these are all well-known signature attributes of the FEF (Robinson and Fuchs 1969; Bruce et al. 1985; Schall 1991).

\section{General protocol of infusion experiments}

As an overview, each experiment involved the following general sequence of events. A microelectrode and needle were lowered together into the left FEF until an acceptable multiunit site was found. The monkey was run on a task, providing "before" FEF inactivation data. Lidocaine, muscimol, or saline was then infused through the needle, and "during" FEF inactivation data was collected. Following recovery, "after" data were collected.

\section{Infusion methods}

We infused lidocaine (lidocaine hydrochloride, $2 \%$ solution; Steris Laboratories, Phoenix, Ariz., USA), muscimol (5-aminomethyl-3hydroxyisoxazole; Sigma, St. Louis, Mis., USA), or saline at a site while monitoring the nearby neural activity. The cut end of a 30 gauge needle was epoxied into one end of a 28 gauge cannula, so that $16 \mathrm{~mm}$ of the needle was exposed. PE 50 tubing was fit snugly over the other cannula end. About $80 \mathrm{~cm}$ of tubing was run to a $100-\mu l$ Hamilton syringe fixed in a slow injector. The tubing was filled with distilled water except for the pharmacological agent at the needle end; a bubble separated the two liquids. A hydraulic microdrive assembly held the needle in parallel with a recording microelectrode (Pt-Ir, glass-coated, $\sim 1.0 \mathrm{M} \Omega$ at $1 \mathrm{kHz}$ ) so that they moved in concert through the dura, into the brain, with their tips $1.5 \mathrm{~mm}$ apart.

For monkey L, the low-threshold, $<50 \mu \mathrm{A}$, sites for evoking saccades were 3-6 $\mathrm{mm}$ below the first recorded unit(s). Thus, we 
Fig. 1 Penetration sites for frontal eye field (FEF) infusion. Top: a lateral oblique view of a rhesus monkey brain. Each circle represents the FEF chamber for a monkey. Below: for monkey I (left) and monkey $\mathrm{L}$ (right), the FEF infusion sites are shown relative to the principal sulcus $(P s)$ and arcuate sulcus $(A s)$. Sulcal locations were determined from histology, for monkey L, and by inspection through the dura, in surgery, for monkey I. The current threshold for evoking saccades was $<50 \mu \mathrm{A}$, for at least one depth, for every one of these 14 sites. Key lists the symbols used to designate which tasks were run during experiments at each site and which pharmacological agents were infused. Concentric circles at a site depict the number of experiments done at the site, with inner circles representing earlier experiments. Lower-case letters beside the symbol for each experiment provide labels for associating experiments with penetration locations

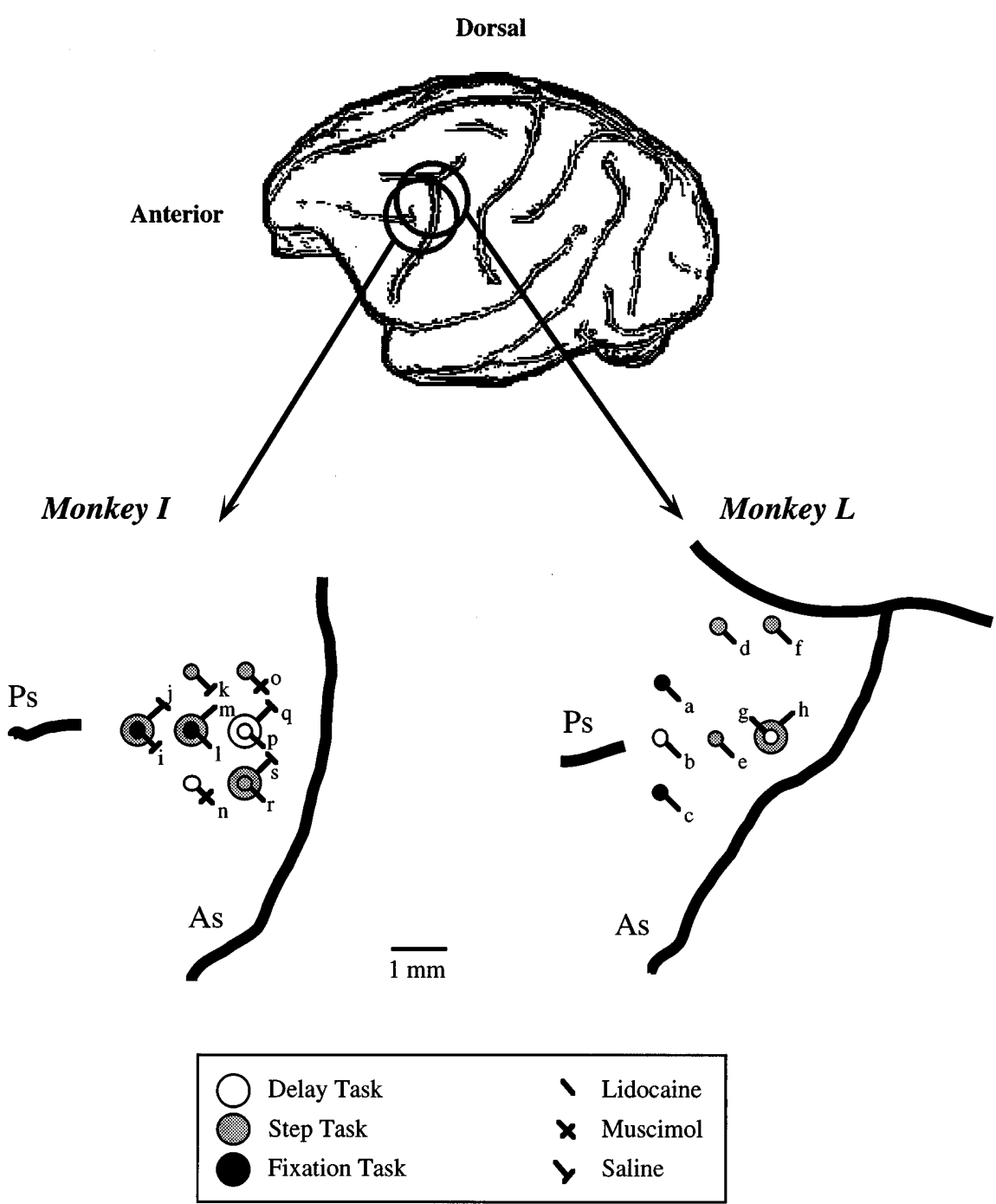

placed the needle and electrode tips within this depth range during each infusion experiment involving monkey L. For monkey I we placed the needle and electrode tips 4-7 $\mathrm{mm}$ below the first unit(s), for the same reasons. Within the appropriate depth range, we found a site with reasonably high $(>5 \mathrm{~Hz})$ and robust multiunit activity, so that neural inactivation could be confidently monitored.

For lidocaine infusion, $18 \mu \mathrm{l}$ of the $2 \%$ solution was infused at $4 \mu \mathrm{l} / \mathrm{min}$. This has been shown to inactivate units $1.5 \mathrm{~mm}$ away, i.e., at the recording electrode, with nearly $100 \%$ certainty (Tehovnik and Sommer, 1997).

For muscimol infusion, the apparatus was modified: a $25-\mu \mathrm{l}$ Hamilton syringe, providing $0.5 \mu \mathrm{l}$ resolution, was used instead of the $100-\mu \mathrm{l}$ one. Muscimol was dissolved in saline to $2 \mu \mathrm{g} / \mu \mathrm{l}$. We infused $2 \mu \mathrm{l}$ of the solution over $13.5 \mathrm{~min}$. These parameters were selected to be similar to those used previously (Hikosaka and Wurtz 1985; Schiller et al. 1987; Munoz and Wurtz 1993; Robinson et al. 1993; Kurata and Hoffman 1994; Ohtsuka et al. 1994).

\section{Data collection}

Experiments were controlled by a PDP-11 computer. The microelectrode signal was amplified (BAK, A-1B), spikes were discriminated (BAK, DIS-1), and Schmitt trigger signals corresponding to the spikes were sent to the PDP-11. Data files recorded eye position (sampled at $333 \mathrm{~Hz}$ ), task events, and the mean firing rate during each trial.

\section{Visual stimulation}

Sixty-three yellow light-emitting diodes (LEDs; $18 \mathrm{~cd} / \mathrm{m}^{2}$ ) were fixed in a board that was curved horizontally and vertically to point the LEDs at the monkey, minimizing differences in luminance caused by angle. The monkey sat $108 \mathrm{~cm}$ from the board. The LEDs were arranged in 7 rows of 9 , spaced by $5 \mathrm{deg}$ in the cardinal directions, for a total area of $40 \mathrm{deg}$ horizontally by 30 deg vertically. Subsets of the LEDs were used as targets or fixation points in an experiment. Prior to each experiment we calibrated the eye position signal by having the monkey look at LEDs illuminated for several seconds in various positions on the board.

Except for the glow caused by LEDs when lit, the testing room was in darkness. The use of darkness as background allowed for precise control over photic stimulation and maximized target contrast, optimizing the monkey's ability to see the LEDs. The room light and the entire array of LEDs were turned on for several seconds between blocks of trials to keep the monkey alert. Occasionally the monkey was given breaks in the light for 10-20 min to prevent drowsiness.

\section{Oculomotor tasks}

Three tasks were used (Fig. 2): the delay task, the step task, and the fixation task. A single task was used in each testing session. A block of trials lasted 8 to 30 min, depending on the monkey's ability to perform the task during FEF inactivation. Juice reward was 


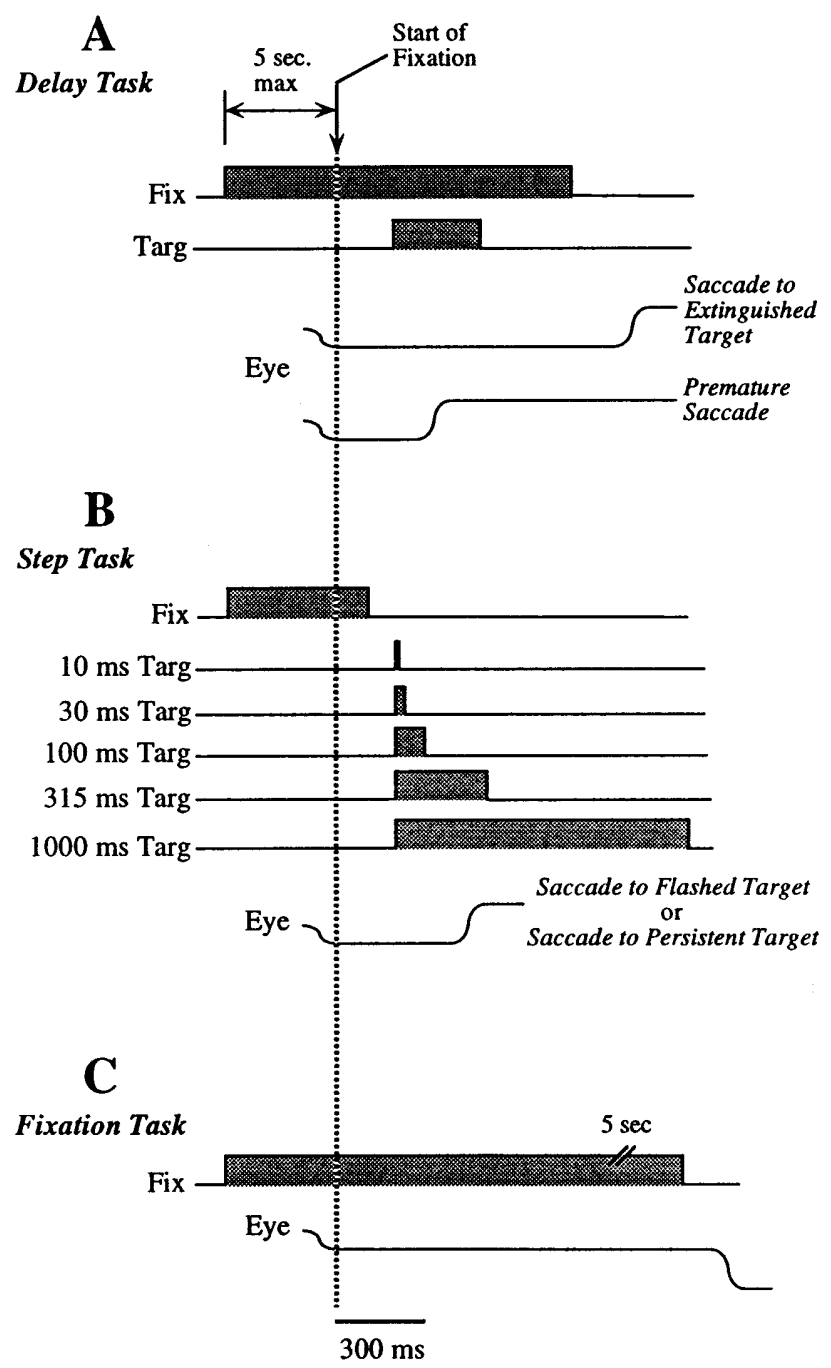

Fig. 2A-C Timing of the tasks. In each task, the monkey initially had $5 \mathrm{~s}$ to acquire the fixation light-emitting diode (LED) (top). Once fixation began, the remaining events occurred. A Delay task. After foveation of the fixation LED (Fix) a target LED (Targ) was lit and then was extinguished. The monkey was required to maintain fixation until the fixation LED disappeared, and then it was allowed to make a saccade to the location of the extinguished target (Eye, top trace). A saccade was premature if it was initiated before fixation offset (Eye, bottom trace). B Step task. After fixation, the fixation LED (Fix) disappeared, there was a brief gap with no stimuli present, and then a target LED was lit. The monkey was allowed to go to the target as soon as it appeared (Eye trace). Flashed targets were of 10,30 , or $100 \mathrm{~ms}$ duration and persistent targets were of $1000 \mathrm{~ms}$ duration. An intermediate, $315 \mathrm{~ms}$ duration, target also was used. C Fixation task. After initial fixation of an LED (Fix), the monkey was required to maintain its eye position near it until it disappeared. Lower trace (Eye) shows correct performance. Time scale is at the bottom

given for correct responses, and in cases of severe deficits, incorrect responses sometimes were rewarded to keep the animal from quitting.

The computer judged on-line that the monkey was looking at an LED if two conditions were met: the eye position was within an electronic window around the LED position and a fixation began, i.e., the eye velocity fell below $50 \mathrm{deg} / \mathrm{s}$. As soon as an initial fixation began, the sequence of other trial events was initiated (Fig. 2), and as soon as a correct saccade ended, reward was delivered.
Because there is an inevitable upwards drift and a compromise in accuracy for saccades made in darkness to the locations of extinguished targets in delay tasks (Gnadt et al. 1991; White et al. 1994), target windows were 10 deg horizontally by 20 deg vertically and fixation windows were $10 \times 10 \mathrm{deg}$ square. The same window sizes were used in all our tasks to keep conditions as similar as possible between experiments.

\section{Delay task}

A fixation LED appeared and was foveated (Fig. 2A). After $200 \mathrm{~ms}$, a target LED appeared for $300 \mathrm{~ms}$ and then disappeared. After a $300 \mathrm{~ms}$ delay period, the fixation LED disappeared: this was the cue to move. The monkey then had $2 \mathrm{~s}$ to move. A correct response was a single saccade that landed in the target window. If a saccade was made before the cue to move, it was "premature". If a saccade was made before target onset, the trial was aborted. Twenty or more target locations and three initial fixation positions (20 deg ipsilateral, central, and 20 deg contralateral) always were randomized by trial.

\section{Step task}

A fixation LED was lit to start a trial and, $100 \mathrm{~ms}$ after the monkey foveated it, it disappeared (Fig. 2B). After $100 \mathrm{~ms}$, a target LED was lit. The monkey then had $2 \mathrm{~s}$ to move. A correct response was a single saccade that landed in the target window. If a saccade was made before target onset, the trial was aborted. Twenty or more target locations always were randomized by trial. Additionally, in some experiments, three initial fixation positions (20 deg ipsilateral, central, or 20 deg contralateral) were randomized by trial, and target duration was set to either 30 or $1000 \mathrm{~ms}$. In other experiments, the target duration was randomized by trial $(10,30,100,315$, or $1000 \mathrm{~ms})$ and only central fixation was used. The 10,30 , and $100 \mathrm{~ms}$ duration targets were termed "flashed" because they always appeared and disappeared before a saccade began. The $1000 \mathrm{~ms}$ duration targets were termed "persistent" because they were still lit after a saccade was made in more than 99\% of trials. The $100 \mathrm{~ms}$ "gap" of darkness before target onset was used to encourage the production of express saccades (Schiller et al. 1987).

\section{Fixation task}

The monkey waited in darkness with eye position unconstrained, and after a random interval $(\sim 6 \mathrm{~s})$ one LED was illuminated (Fig. 2C). This LED was chosen randomly from an array of 9 or 20 LED locations distributed across the testing space. The monkey had $5 \mathrm{~s}$ to acquire the LED, i.e., fixate within the $10 \times 10 \mathrm{deg}$ window around it, and then it had to keep its eye position within the window for an additional $5 \mathrm{~s}$.

\section{Analysis}

We analyzed the first saccade made after target onset. Trials in which the first saccade was $\leq 2.0$ deg in amplitude (within the range of amplitudes of fixation-related microsaccades in our monkeys) and trials in which no saccade was detected were pooled as "No Saccade" trials. These trials were counted but were not analyzed further. For trials in which the first saccade was $>2.0$ deg in amplitude, the saccade was characterized as follows. The saccadic error was defined as the vectorial distance from the saccade's endpoint to the target's location. The saccadic latency was defined as the time to saccade initiation after either (1) fixation spot disappearance, for saccades made after the cue to move in the delay task, or (2) target onset, for premature saccades in the delay task and all saccades in the step task. Saccadic peak velocity and amplitude were measured for a consideration of dynamics. Second- 
ary, corrective saccades were rare and were not analyzed. Aborted trials were discarded.

Monkeys were run on tasks continuously for about $2 \mathrm{~h}$ after a lidocaine infusion (except during rest breaks) and periodically, every $30 \mathrm{~min}$ or so, after a muscimol infusion. Out of all the eye movement data, three sets, called "before", "during", and "after", were fully quantified and compared. "Before" data were those collected just before infusion. For lidocaine experiments, "during" data were those collected 5-30 min after the start of the infusion (while FEF units were inactivated) and "after" data were the last data collected that day. For muscimol experiments, "during" data were those collected approximately $90 \mathrm{~min}$ after the start of the infusion (while FEF units were inactivated), and "after" data were collected the next day. For saline experiments, FEF inactivation did not occur, so "during" and "after" data sets were time-matched to the "during" and "after" data sets of lidocaine experiments (for saline controls of lidocaine volume) or muscimol experiments (for saline controls of muscimol volume).

We tested hypotheses that the "during" or "after" data were indistinguishable from the "before" data, using the unpaired, twotailed Student's $t$-test with Bonferroni correction (traditional $P$ level was halved, to 0.025). Impairments affected entire hemifields, so contralateral target trials were pooled and ipsilateral target trials were pooled. Trials with targets on the vertical meridian were not statistically analyzed because of small sample size. To test hypotheses that changes in percentages were significant, chisquare or Fisher exact tests were used, as appropriate, with Bonferroni correction.

For the remaining analyses, Bonferroni correction was not required (significance criterion was $P<0.05$ ). To determine whether saccadic error covaried with saccadic latency during FEF inactivation, the Pearson correlation coefficient was found. To determine whether eye position in the dark shifted during FEF inactivation, the paired, two-tailed Student's $t$-test was used.

\section{Histology}

Monkey L was overdosed with pentobarbital, perfused with $0.9 \%$ $\mathrm{NaCl}$, and fixed with $4 \%$ para-formaldehyde. To estimate the location of injection sites, guide pins were inserted into the cortex at reference locations in the recording chamber. Monkey I is being used for additional experiments.

\section{Results}

Overall infusion results

Nineteen infusions were made into the left FEFs of the monkeys (Fig. 1): 12 were lidocaine infusions, two were muscimol infusions, four were infusions of saline to match the lidocaine volume, and one was an infusion of saline to match the muscimol volume. Results from the two monkeys were similar in all tasks. Results did not appear to vary with injection location. Lidocaine and muscimol infusions caused the same types of effects, but with different time-courses: lidocaine's effects began
Fig. 3 Saccadic deficits of FEF inactivation using the delay task. Saccadic eye movements (dotted curves) are shown before (left), during (center), and after (right) FEF inactivation. Small squares show saccadic endpoints. Small crosses (mostly obscured) show initial fixation locations. Larger squares with dotted outlines represent the 20 target locations. Upper panels show saccades initiated after the cue to move, made in attempt to reach locations of extinguished targets. Lower panels show premature saccades. Data marked with labelled arrows are discussed in the text. Bottom: the mean multiunit firing rate $1.5 \mathrm{~mm}$ from the infusion site is plotted versus time, with 0 representing the end of the infusion. The average firing rate during every trial was plotted and then adjacent data were connected with straight lines. At very bottom, the capital letter identifying the monkey is followed by a parenthesized letter designating the penetration site (see Fig. 1); this is a convention for the entire paper
Before

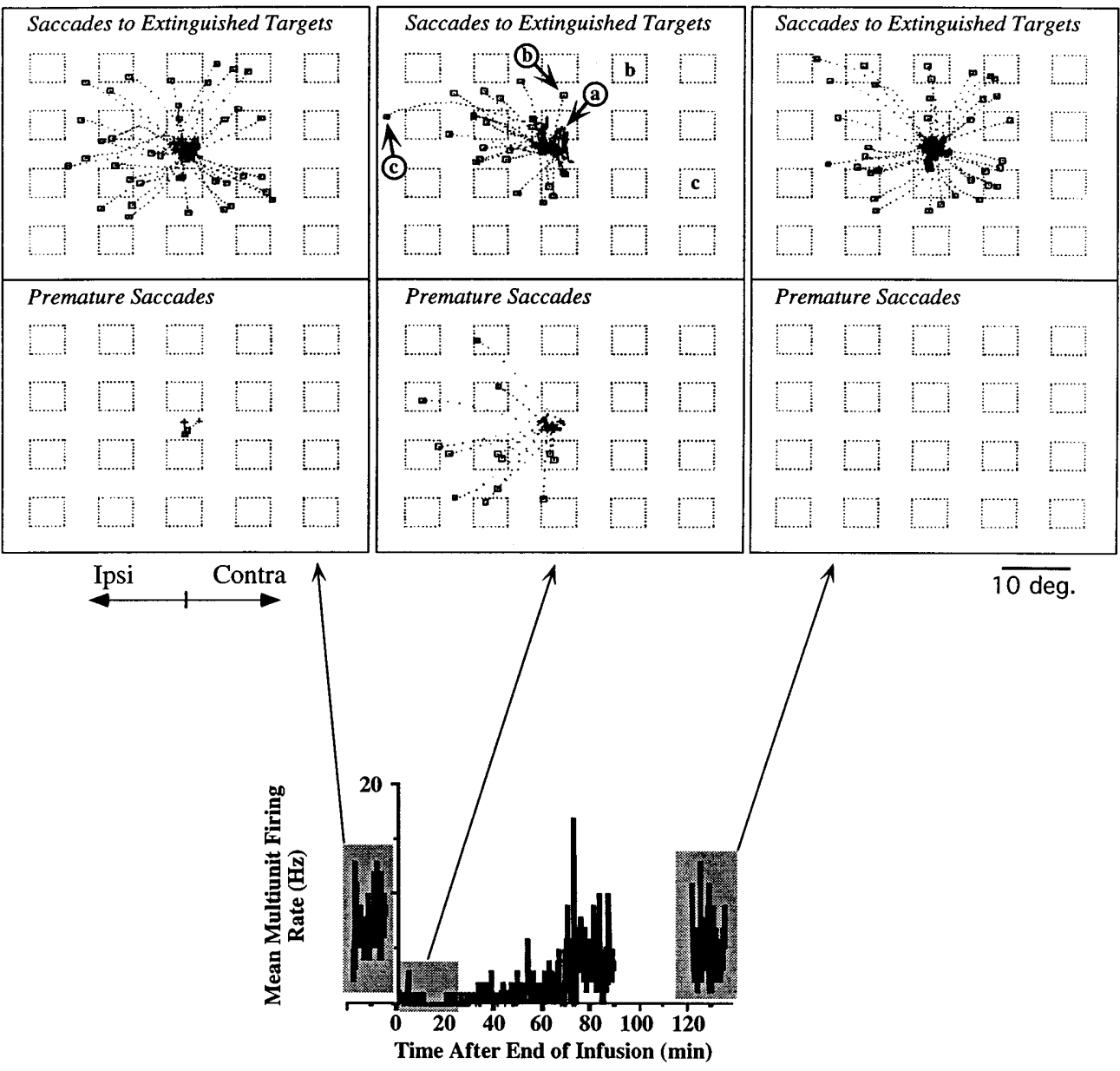



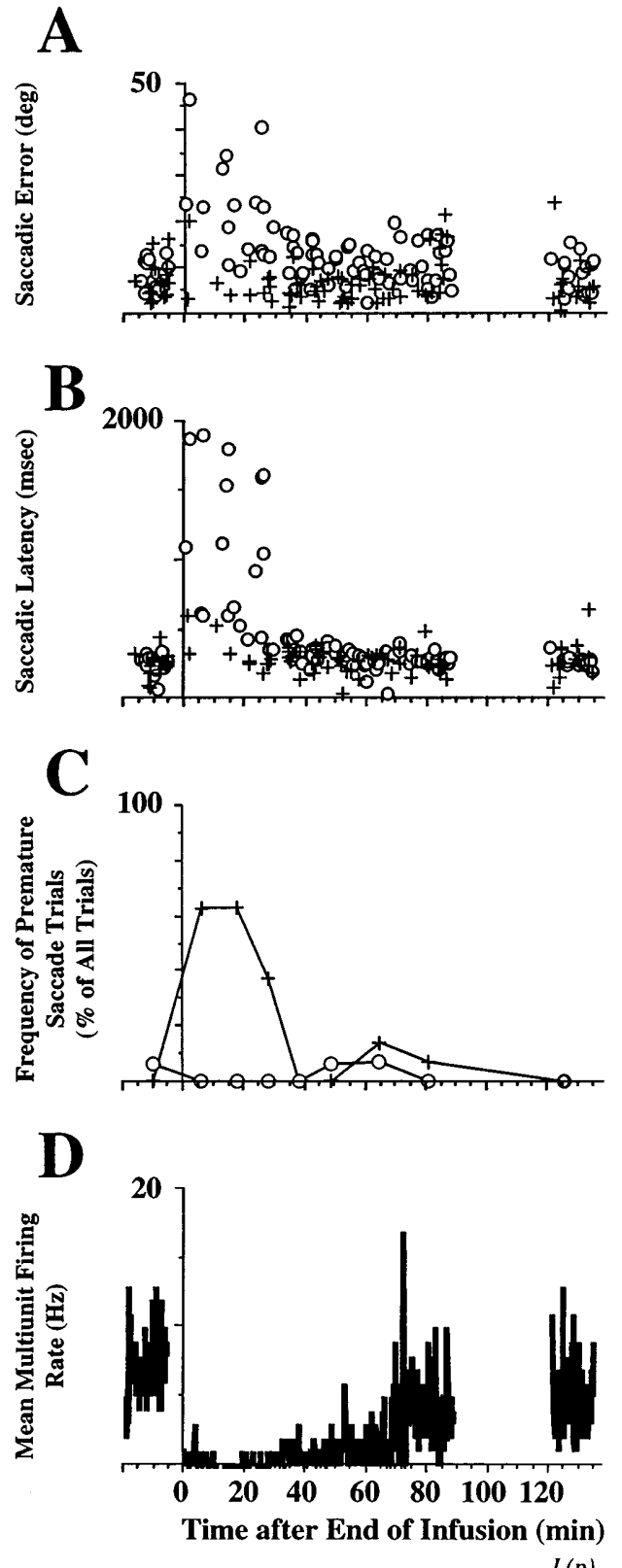

$I(p)$

Fig. 4A-D Trial-by-trial account of saccadic impairment during a delay task experiment. Same experiment as in Fig. 3. Symbols represent saccades made in response to contralateral targets (circles) or ipsilateral targets (crosses). The A error and $\mathbf{B}$ latency of each saccade made after the cue to move, in attempt to reach the location of an extinguished target, are shown. C The frequency of trials in which a premature saccade was made. D Time-course of neural activity $1.5 \mathrm{~mm}$ from the infusion site

within min and lasted about $1 \mathrm{~h}$, but the effects of muscimol began later (about $1 \mathrm{~h}$ after infusion) and lasted longer (recovery did not occur until the next day).

\section{Delay task}

The delay task was used during three lidocaine infusions, one muscimol infusion, and one saline infusion. FEF in-
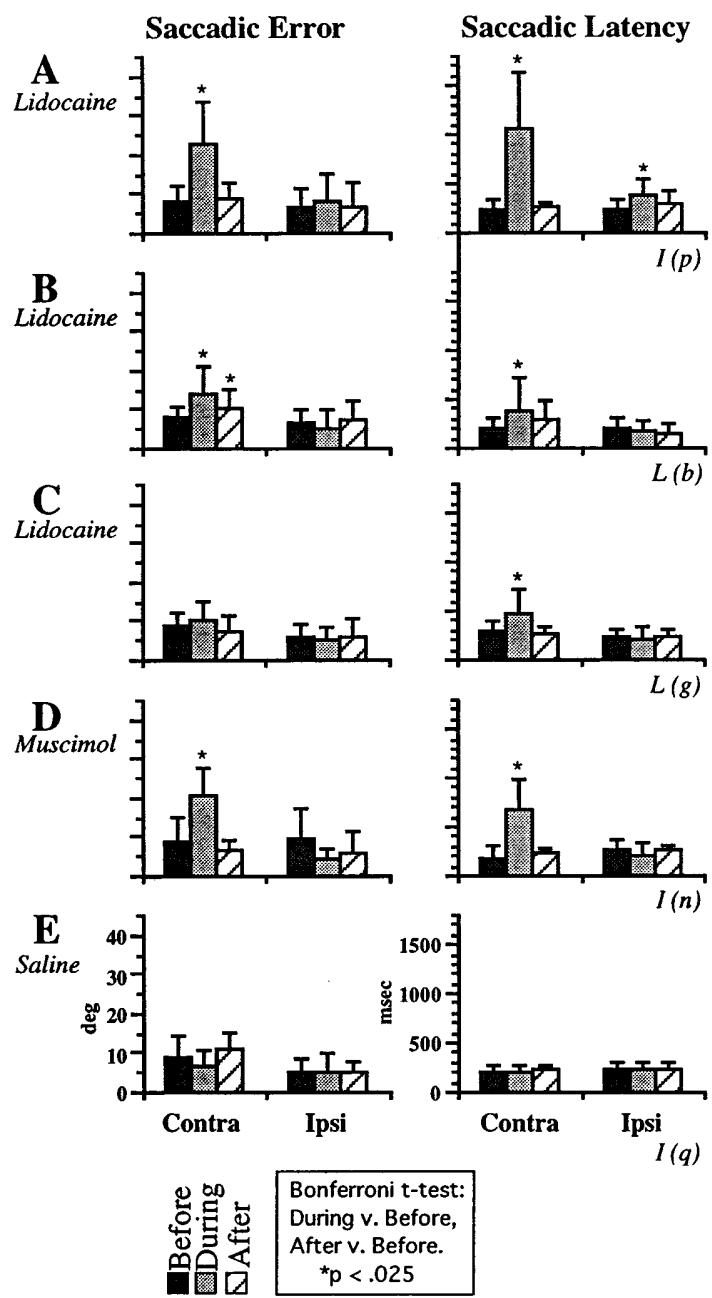

Fig. 5A-E Quantification of saccadic error and latency for all delay task experiments. Mean and standard deviation of error (left column) and latency (right column) are shown before, during, and after FEF inactivation for saccades made after the cue to move, in attempt to reach locations of extinguished contralateral or ipsilateral targets. A Results of the lidocaine infusion from Figs. 3 and 4. B,C Results of lidocaine infusions from the other monkey. D Results of the muscimol infusion. E Results of the saline infusion. Degrees of freedom ranged from 20 to 76

activation severely impaired the generation of saccades to locations of extinguished contralateral targets and it promoted the generation of premature saccades to ipsilateral targets.

\section{Results from central fixation}

In Fig. 3, a monkey's eye movements are shown before, during, and after left FEF inactivation. Before the infusion, the monkey made saccades toward all locations of extinguished targets (Fig. 3, Before). As is typical of saccades made in darkness to locations of extinguished targets, these saccades tended to be hypometric and biased upwards (Gnadt et al. 1991; White et al. 1994). Premature saccades were rare before the infusion (Fig. 3, Before). 
A

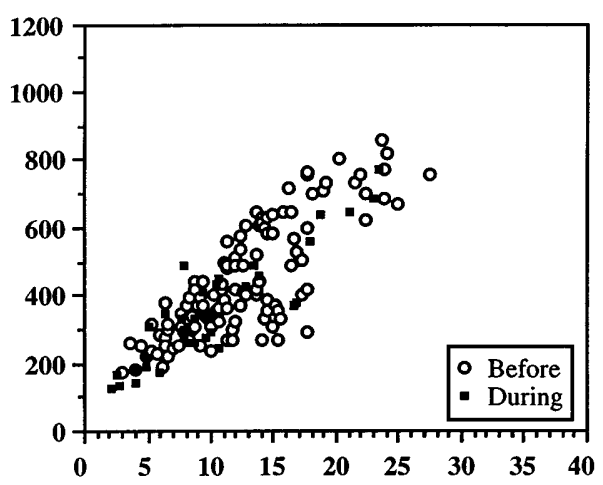

B

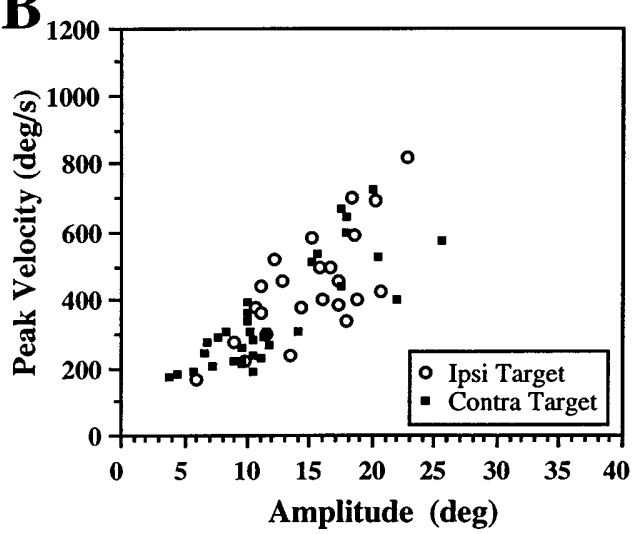

$\mathbf{C}$

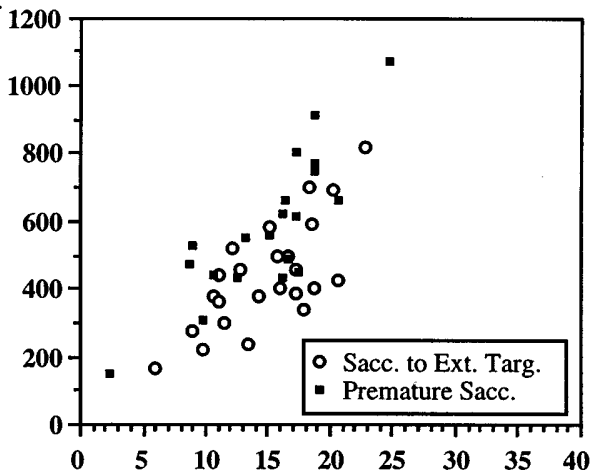

D

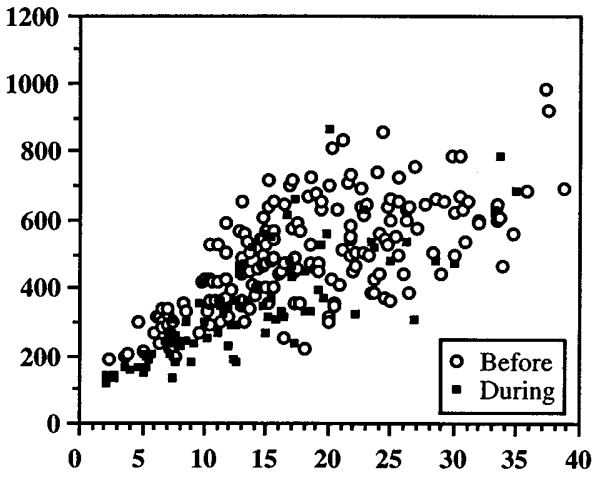

Fig. 6A-D Graphs of peak velocity versus amplitude ("main sequences") for saccades made in the delay task. Data from the three lidocaine experiments and the muscimol experiment are pooled. Symbol explanations are shown in a box in each plot. A Main sequences of contraversive saccades made after the cue to move, in attempt to reach locations of extinguished contralateral targets: saccades made before or during FEF inactivation are compared. B Main sequences of ipsiversive saccades made after the cue to move, during FEF inactivation: saccades made in attempt to reach locations of extinguished contralateral or ipsilateral targets are compared. C Main sequences of saccades made to locations of ipsilateral targets during FEF inactivation: premature saccades are compared with saccades made after the cue to move. D From the experiments using initially ipsilateral fixation, main sequences of contraversive saccades made after the cue to move, in attempt to reach locations of extinguished contralateral targets, are shown: saccades made before or during FEF inactivation are compared

Multiple units recorded $1.5 \mathrm{~mm}$ away from the infusion site had a mean firing rate of approximately $8 \mathrm{~Hz}$ (Fig. 3, bottom). Following lidocaine infusion (time 0 ), the firing rate dropped and saccades were impaired (Fig. 3, During). Out of the 16 trials in which contralateral targets were presented during FEF inactivation (eight targets, each presented twice), the following responses occurred. In four trials, a steady fixation or a drift occurred instead of a saccade (examples labelled "a", Fig. 3, During). In five trials, contraversive saccades were made after the cue to move, but they were very inaccurate (see saccade and its target labelled "b", Fig. 3, During). In the remaining seven trials, ipsiversive saccades were made after the cue to move (see saccade and its target labelled "c", Fig. 3, During). These ipsiversive saccades headed in the wrong

A
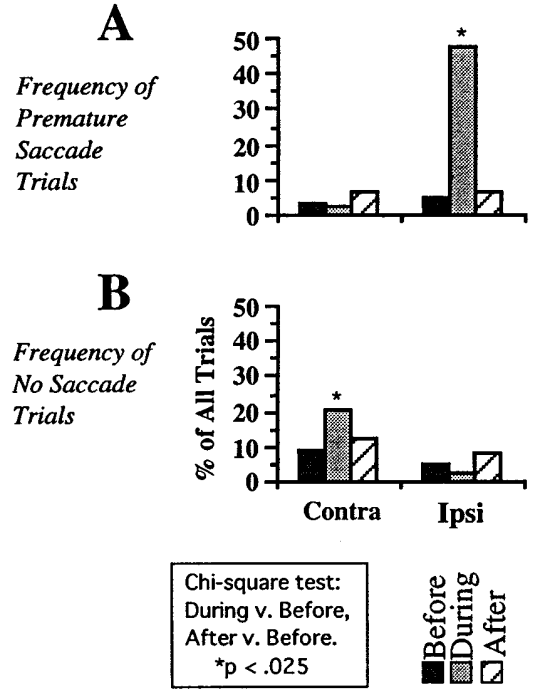

Fig. 7A, B Frequencies of trials in which saccades were made prematurely or not at all in the delay task. Results of the three lidocaine experiments and the muscimol experiment are pooled. A The percentage of trials in which a premature saccade was made. B The percentage of trials in which no saccade was made

direction even from their moment of initiation. Out of the 16 trials in which ipsilateral targets were presented, saccades always went ipsiversively and their accuracies were normal, but in ten trials the saccades were premature. Finally, in the 8 trials in which targets were presented on the vertical meridian, in three trials saccades were premature and went vertically. In the other five trials, saccades 
Fig. 8A, B The effects of FEF inactivation on saccades made in the delay task when initial fixation was A 20 deg ipsilateral or B 20 deg contralateral. Same experiment as in Fig. 3.

Saccades made after the cue to move, in attempt to reach locations of extinguished targets, are shown in upper panels of $\mathbf{A}$ or B. Premature saccades are shown in lower panels of $\mathbf{A}$ or B. Data collected before, during, and after FEF inactivation are shown. Same time periods with respect to neural inactivation as shown in Fig. 3

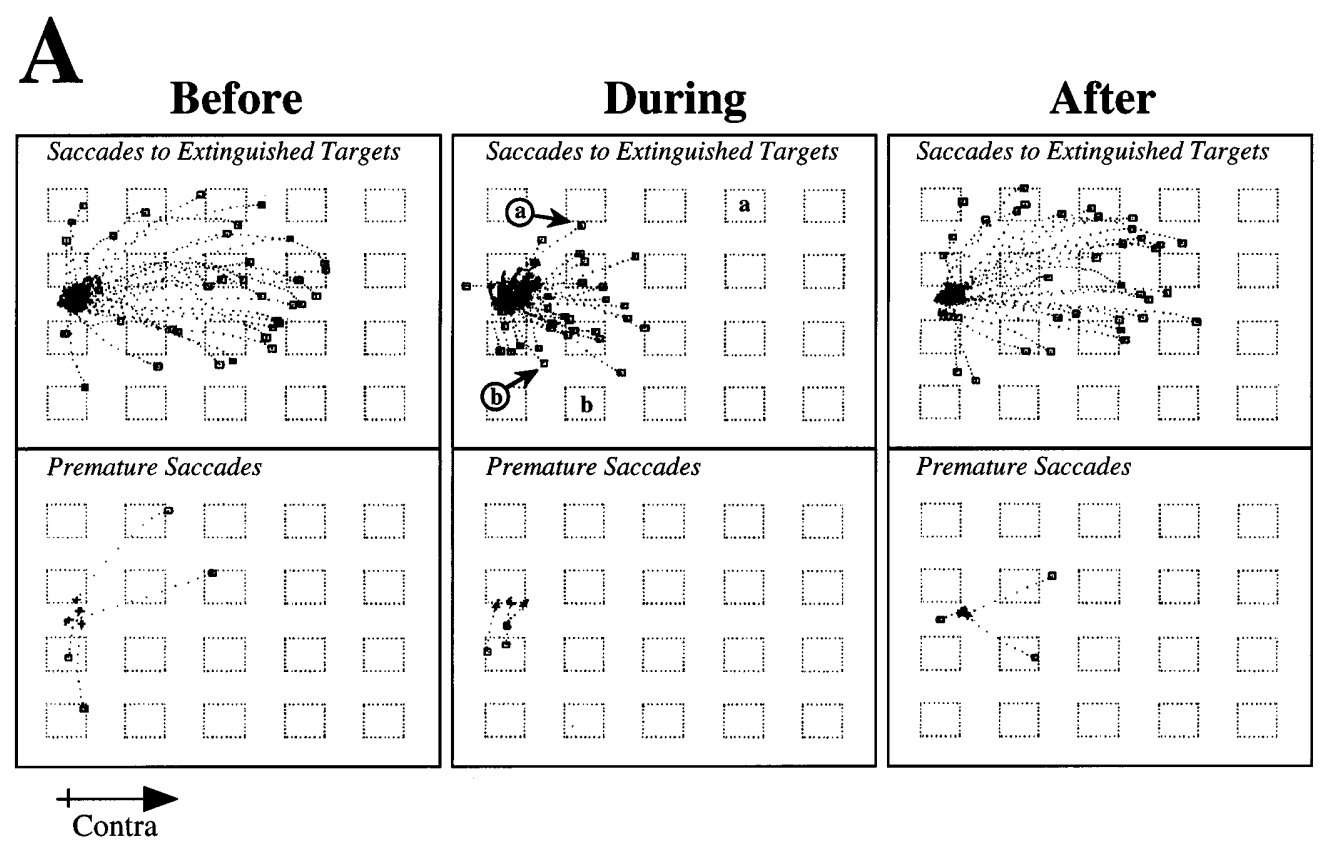

B
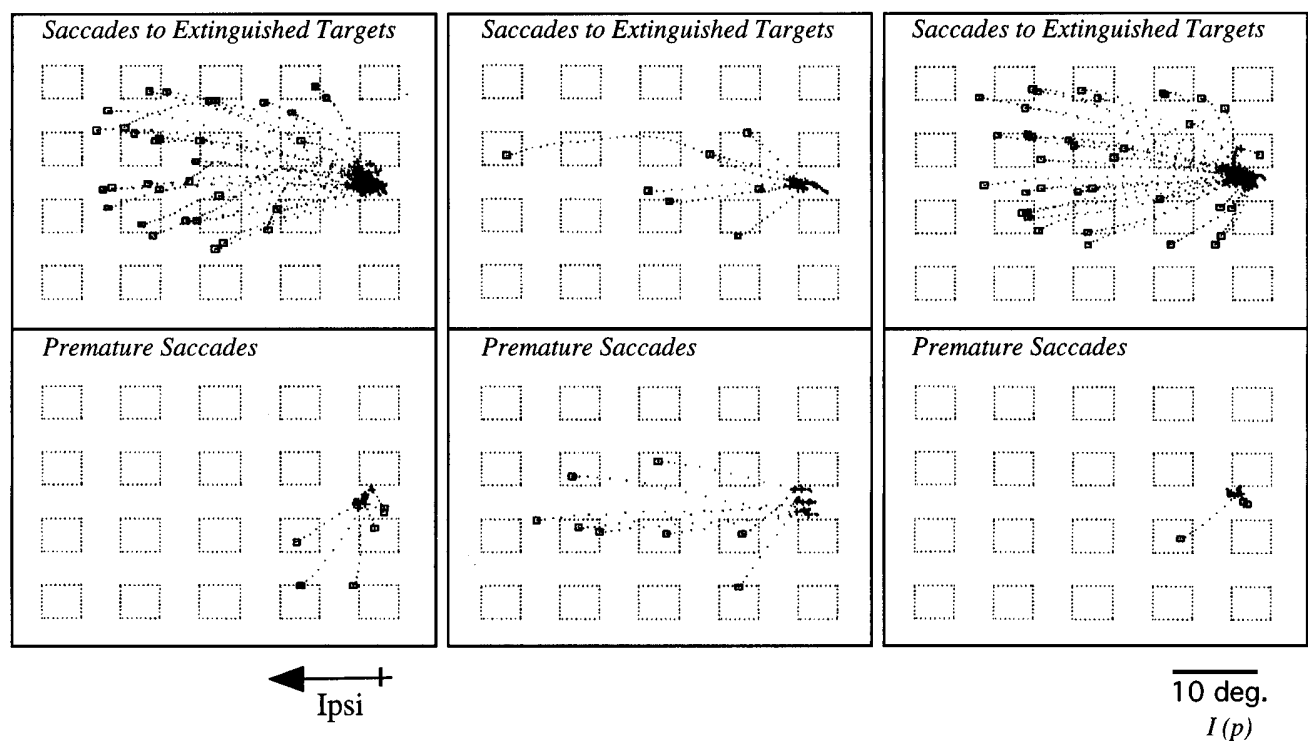

The error and latency results for saccades made after were delayed until after the cue to move and went vertically or ipsiversively. Two hours after the infusion, the neural firing (Fig. 3, bottom) and the behavior (Fig. 3, After) had almost completely recovered.

Figure 4 quantifies the continual time-courses of effects for the same experiment. Saccades made after the cue to move, in attempt to reach locations of extinguished contralateral targets, exhibited increased errors (Fig. 4A) and increased latencies (Fig. 4B) just after the end of the infusion. These effects lasted about $30 \mathrm{~min}$, while FEF activity was at its lowest level (Fig. 4D). Also, the rate of making premature saccades to ipsilateral targets was elevated during this time (Fig. 4C). Behavioral recovery over time (Fig. 4A-C) roughly paralleled the neural recovery (Fig. 4D). the cue to move are shown for all the delay task experiments in Fig. 5. Saccadic error increased significantly for contralateral target trials during FEF inactivation in three of four experiments (Fig. 5A-D, left, shaded vs dark bars). Saccadic latency increased significantly for contralateral target trials during all four FEF inactivations (Fig. 5A-D, right, shaded vs dark bars). The only change in ipsilateral target trials was an increase in saccadic latency during one FEF inactivation (Fig. 5A, right). After FEF inactivation, saccadic error and latency usually recovered (hatched vs dark bars). Infusion of saline to match the lidocaine volume caused no effects (Fig. 5E).

Overall, saccades made after the cue to move, in response to contralateral targets, were directed contra- 
Fig. 9 Saccadic deficits of FEF inactivation using the step task with random target durations. Conventions as in Fig. 3. Top row: saccades made in response to $10 \mathrm{~ms}$ duration flashed targets. Middle row: saccades made in response to $100 \mathrm{~ms} \mathrm{du}-$ ration flashed targets. Bottom row: saccades made in response to $1000 \mathrm{~ms}$ duration persistent targets. Saccades made before, during, and after FEF inactivation are shown

\section{Before}
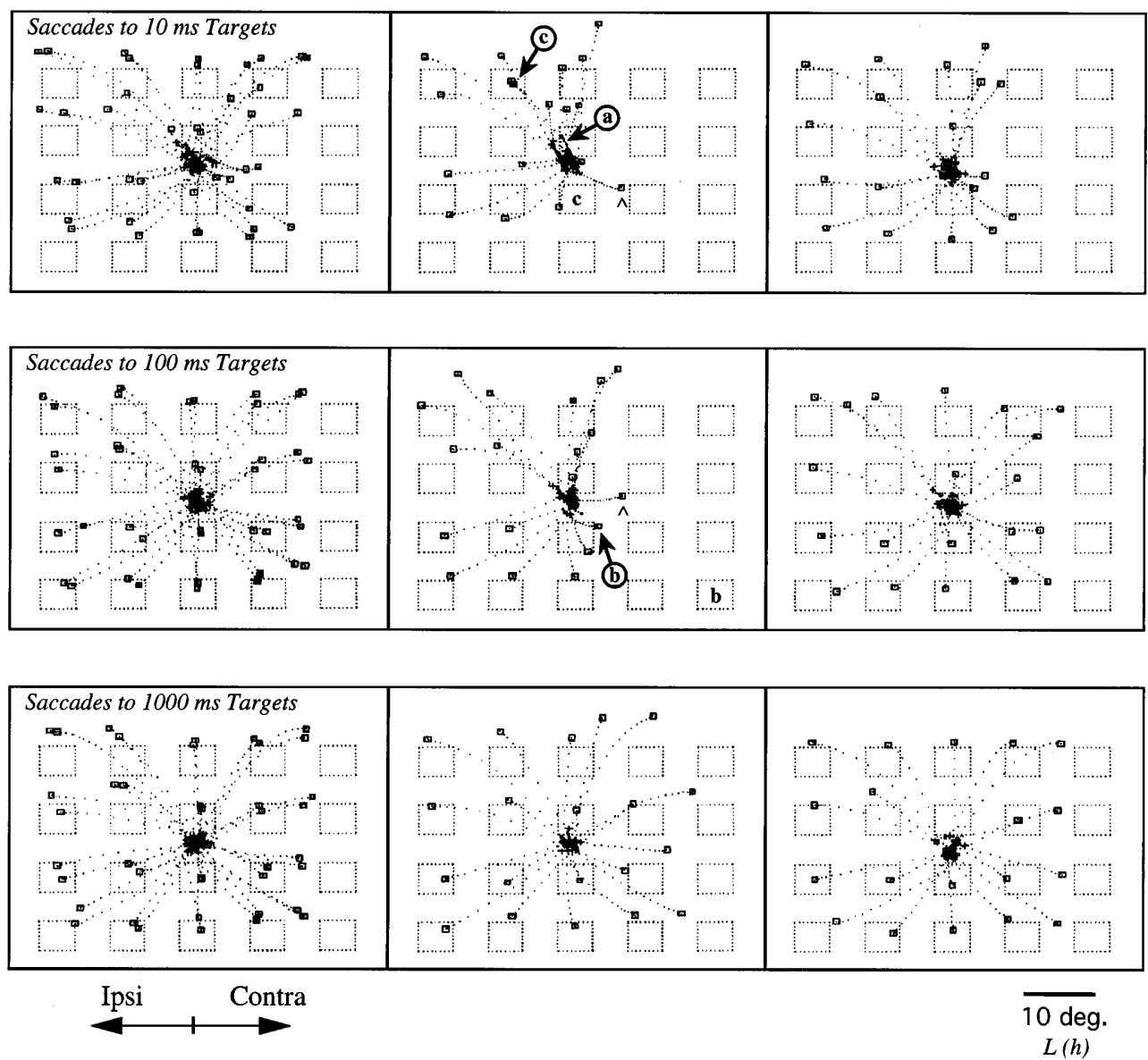

versively only half the time during FEF inactivation (34/68 saccades, experiments of Fig. 5A-D pooled). Peak velocities of these saccades were not affected appreciably by FEF inactivation (Fig. 6A). The ipsiversive saccades made in the other half of the contralateral target trials had peak velocities similar to those of saccades made in response to ipsilateral targets (Fig. 6B).

An increased frequency of making premature saccades to ipsilateral targets during FEF inactivation was found in every delay task experiment. Overall, premature saccades were made in $48 \%$ of ipsilateral target trials during FEF inactivation (Fig. 7A), greatly exceeding the baseline rate of $5 \%$. Premature saccades had a mean latency with respect to target onset of $351 \mathrm{~ms}$ (SD $138 \mathrm{~ms}$ ) and a mean error of $4.0 \mathrm{deg}$ (SD $4.8 \mathrm{deg}$ ). The shortest latency of a premature saccade was $195 \mathrm{~ms}$. Peak velocities of premature saccades tended to exceed those of saccades made after the cue to move (Fig. 6C). This was expected, since saccades made directly in response to a target have higher velocities than saccades made to the location of an extinguished target (Gnadt et al. 1991; White et al. 1994).

During FEF inactivation, the rate of failing to initiate a saccade after the cue to move increased significantly in response to contralateral targets (recall the drifts labelled "a" in Fig. 3, During). "No Saccade" trials occurred $20 \%$ of the time during FEF inactivation when contralateral targets were used (Fig. 7B), up from $9 \%$ of the time before $\mathrm{FEF}$ inactivation.

\section{Results from eccentric fixation}

The above results pertained to the one-third of trials in which initial fixation was central. In another one-third of the trials, initial fixation location was $20 \mathrm{deg}$ ipsilateral. Figure 8A shows the ipsilateral fixation trials for the same experiment as in Fig. 3. During FEF inactivation, almost all the saccades made after the cue to move, in attempt to reach locations of the extinguished contralateral targets, were contraversive (Fig. 8A, During); this ability to make contraversive saccades was in marked contrast to the situation with central fixation (cf. Fig. 3, During). The contraversive saccades made from ipsilateral fixation were abnormally hypometric but their direction was always toward the location of the extinguished target (Fig. 8A, During, saccades and targets labelled "a" and "b" are shown as examples). Other disturbances were similar to those described for central fixation: there were some fixations and drifts instead of saccades and, rarely, there were ipsiversive saccades (Fig. 8A, During). Contraversive saccades made from ipsilateral fixation had nor- 
Fig. 10A-D Quantification of the error and latency of saccades to contralateral targets in the step task experiments that used random target durations. For each target duration, the mean and standard deviation of the saccadic error (left column) and the saccadic latency (right column) are plotted, for before, during, and after FEF inactivation. A Results of the lidocaine experiment of Fig. 9. B Results of the lidocaine experiment from the other monkey. C Results of the muscimol experiment. D Results of the saline experiment. Degrees of freedom ranged from 14 to 30
Saccadic Error

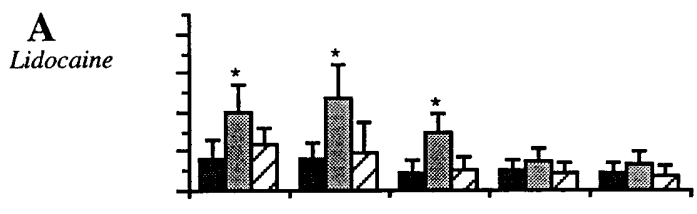

B

Lidocaine

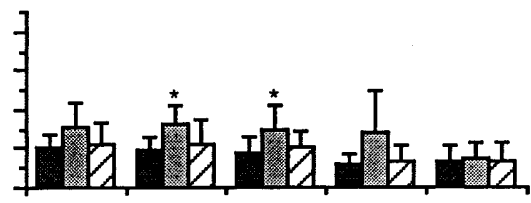

$\underset{\text { Muscimol }}{\text { C }}$
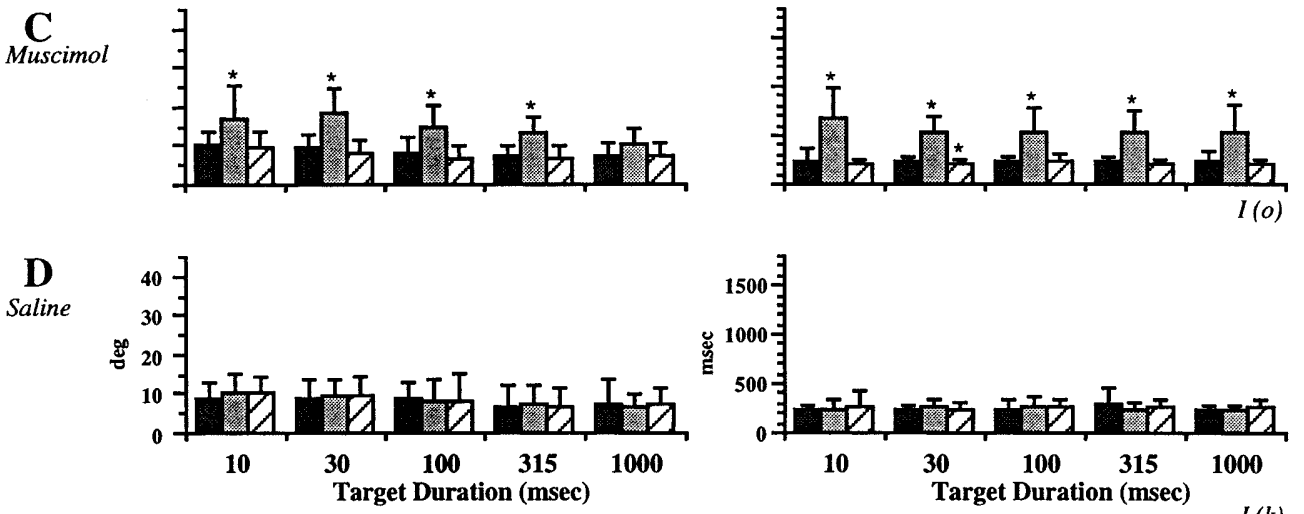

$I(k)$ mal peak velocities during FEF inactivation except for some lowered velocities in the 7-13 deg amplitude range (Fig. 6D). Premature saccades were infrequent from ipsilateral fixation during FEF inactivation (Fig. 8A, During).

In the final one-third of trials, the initial fixation location was 20 deg contralateral (Fig. 8B). During FEF inactivation, monkeys often could not fixate the contralateral LED long enough to allow for target presentation. In the example of Fig. 8B (During), the monkey succeeded in fixating the contralateral LED in only 15 of 40 attempts. Of the 15 trials that were completed, eight resulted in ipsiversive premature saccades (Fig. 8B, During). Saccades were made normally after the cue to move in the other seven trials. They were made even to locations of extinguished targets on the righthand side of the board, i.e., in contralateral head-centered space (Fig. 8B, During). Recall that saccades did not enter this space from central or ipsilateral fixation during FEF inactivation (cf. Fig. 3, During, and Fig. 8A, During).

\section{Step task}

The step task was used during six lidocaine infusions, one muscimol infusion, and three saline infusions. FEF inactivation severely impaired the ability to make sac- cades to flashed contralateral targets. Saccades made to persistent contralateral targets were less affected.

\section{Results using random target durations}

An example of a step task experiment in which target duration was randomized is illustrated in Fig. 9. Before FEF inactivation, saccades were made in all directions (Fig. 9, Before). Saccades made to some contralateral targets were slightly shorter than saccades made to symmetrically located ipsilateral targets (possibly due to cumulative damage caused by the needle penetrations). During left FEF inactivation, saccades made in response to all flashed contralateral targets and some flashed vertical targets were severely disrupted (Fig. 9, During, top and middle panels). Disruptions were similar to those described in the delay task: saccades sometimes were not initiated, being replaced by fixations or drifts ("a"), saccades sometimes were made contraversively but very inaccurately ("b"), and saccades sometimes went ipsiversively ("c"). Two of the saccades made in response to flashed contralateral targets (" $\wedge$ ") were atypical in that they were strongly contraversive with clearly decreased velocities (note the close spacing of their eye position dots). Saccades made in response to persistent contralat- 
Fig. 11A-D Quantification of the error and latency of saccades to ipsilateral targets in the step task experiments that used random target durations. Same experiments as in

Fig. 10: results of $\mathbf{A}$ and $\mathbf{B}$ lidocaine, $\mathbf{C}$ muscimol, and $\mathbf{D}$ saline infusions are shown. Degrees of freedom ranged from 14 to 30
Saccadic Error
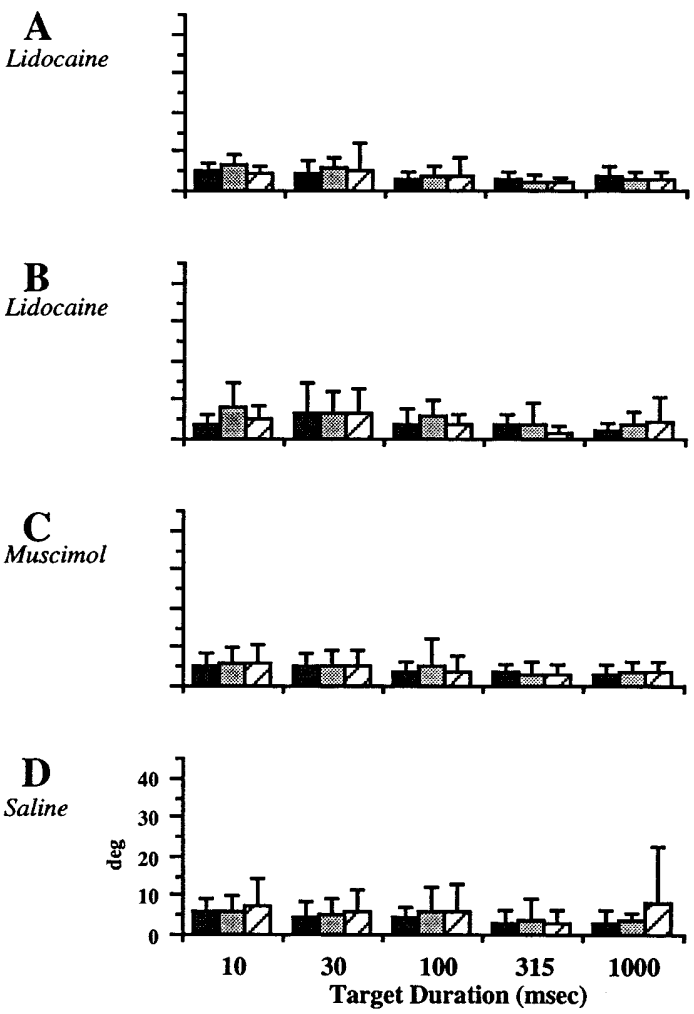

Saccadic Latency
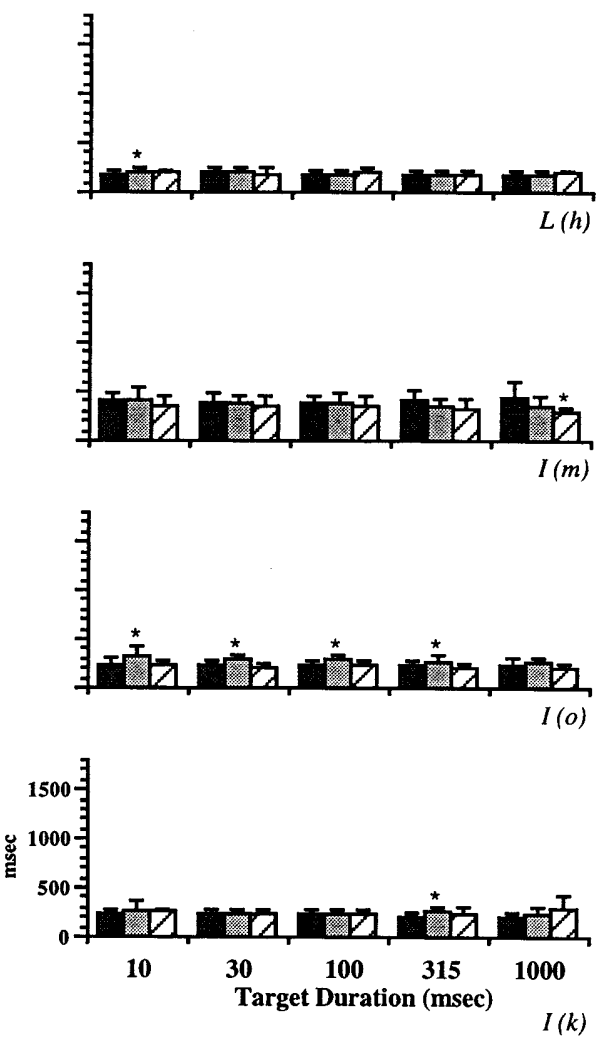

eral targets during FEF inactivation all were contraversive and accurate except for some slightly deviated saccades made to the upper right (Fig. 9, During, bottom). Saccades made in response to all ipsilateral targets were normal during FEF inactivation. After FEF inactivation, the saccadic behavior almost fully recovered (Fig. 9, After), although recovery took longer for saccades made in response to the $10 \mathrm{~ms}$ duration targets.

Figures 10 and 11 quantify the error and latency results of all experiments that used random target durations. Saccades made in response to contralateral targets are quantified in Fig. 10. For the two lidocaine experiments and the muscimol experiment (Fig. 10A-C, left), there was a pattern of increased error for saccades made in response to flashed $(10,30$, or $100 \mathrm{~ms}$ duration) contralateral targets: error was significantly increased during FEF inactivation in eight of the nine cases. For saccades made in response to $315 \mathrm{~ms}$ duration targets, error was significantly increased in one of three cases. For saccades made in response to persistent (1000 ms duration) targets, in no case was the error significantly increased during FEF inactivation.

The effects on saccadic latency were more uniform. In two experiments, latency increased significantly during $\mathrm{FEF}$ inactivation for saccades made in response to every duration of contralateral target (Fig. 10A,C, right).
In one experiment (Fig. 10B, right), latency increased significantly during FEF inactivation for saccades made in response to one of the flashed target durations (100 ms) and to persistent targets (1000 ms duration). In sum, FEF inactivation caused increases in the latencies of saccades made in response to both flashed and persistent contralateral targets.

Saccades made to ipsilateral targets were negligibly affected by FEF inactivation (Fig. 11A-C). In no case did saccadic error change significantly, but sometimes saccadic latency rose slightly. A small latency increase also was seen in the saline infusion, for $315 \mathrm{~ms}$ duration targets (Fig. 11D). This was the only significant change seen in any of the saline infusions.

Although we used a $100 \mathrm{~ms}$ gap of darkness before target presentation, express saccades never were made by our monkeys, either normally or during FEF inactivation. Latency distributions had means of approximately $200 \mathrm{~ms}$ or higher and there was no evidence of the bimodality indicative of express saccade generation (e.g., Sommer 1994). Hence, we were unable to determine whether inactivating the FEF affects express saccades.

Overall, saccades made in response to flashed contralateral targets (10-100 ms duration) during FEF inactivation were contraversive $67 \%$ of the time (58/87 saccades, 
A

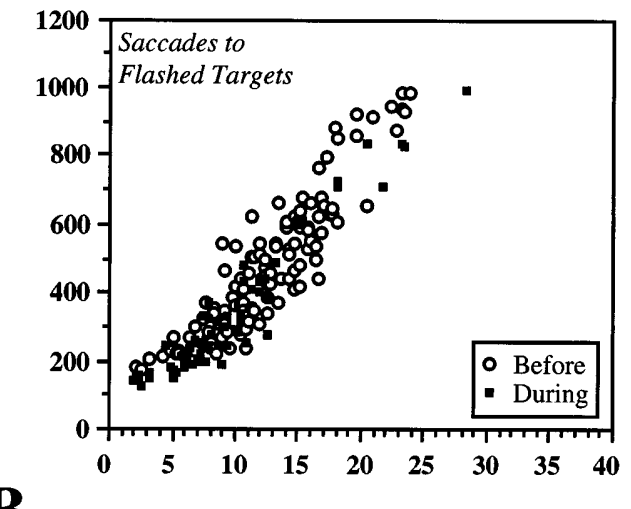

B

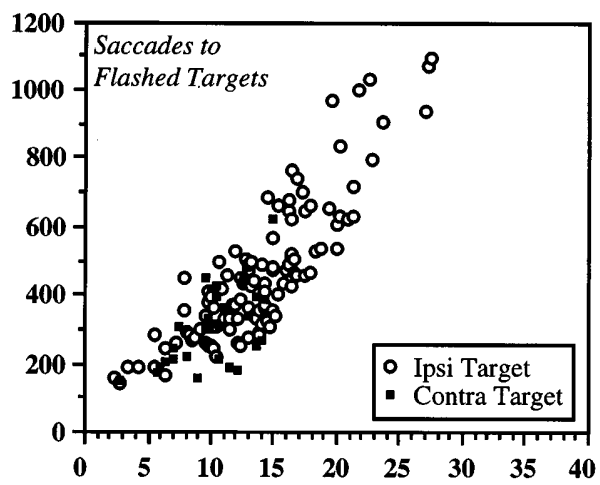

C

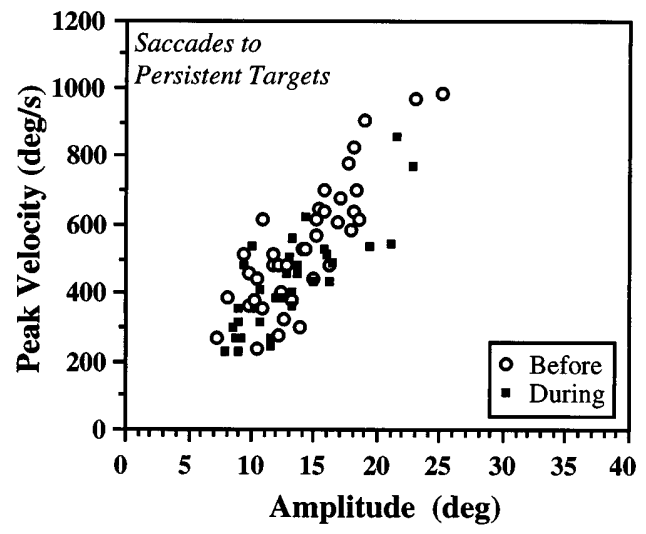

Fig. 12A-C Main sequences of saccades made in the step task with random target durations. Data from the two lidocaine experiments and the muscimol experiment are pooled. Symbol explanations are shown in a box in each plot. A Main sequences of contraversive saccades made in response to flashed contralateral targets: saccades made before or during FEF inactivation are compared. B Main sequences of ipsiversive saccades made in response to flashed targets during FEF inactivation: saccades made in response to contralateral or ipsilateral targets are compared. C Main sequences of contraversive saccades made to persistent targets: saccades made before or during FEF inactivation are compared

experiments of Fig. 10A-C pooled). Peak velocities of contraversive saccades made in response to flashed contralateral targets were unaffected by FEF inactivation, except for saccades in the 2-8 deg amplitude range that tended to have lower velocities (Fig. 12A). Similarly, during FEF inactivation, the ipsiversive saccades made in

\section{A Contralateral Targets}

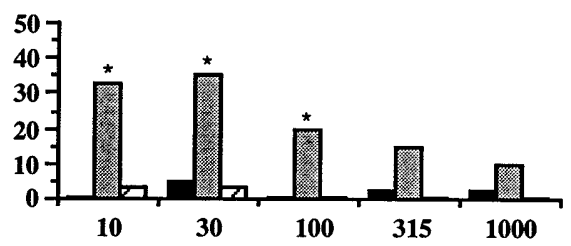

B Ipsilateral Targets
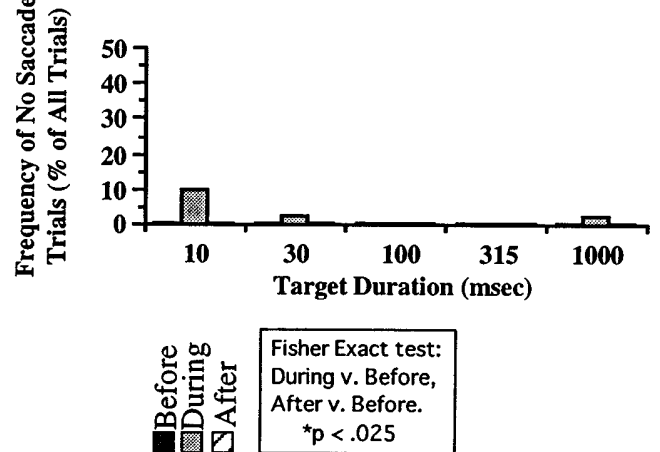

Fig. 13A, B Frequencies of trials in which saccades were not made during the step task experiments with random target durations. Results of the two lidocaine experiments and the muscimol experiment are pooled. The percentage of trials in which no saccade was made in response to $\mathbf{A}$ contralateral targets or $\mathbf{B}$ ipsilateral targets of $10,30,100,315$, or $1000 \mathrm{~ms}$ duration is shown, for before, during, and after FEF inactivation

response to flashed contralateral targets had comparable peak velocities to normal ipsiversive saccades (those made to flashed ipsilateral targets) with a few exceptions of velocity decreases in the 8-13 deg amplitude range (Fig. 12B).

Saccades made in response to persistent contralateral targets (1000 ms duration) were contraversive $97 \%$ of the time (35/36 saccades, experiments of Fig. 10A-C pooled). Their peak velocities were unaffected by FEF inactivation (Fig. 12C).

FEF inactivation often caused a failure to trigger contraversive saccades in the step task. Frequencies of "No Saccade" trials increased significantly for presentation of flashed contralateral targets $(10-100 \mathrm{~ms}$ durations; Fig. 13A). FEF inactivation caused no significant increase in the rate of triggering saccades to longer duration contralateral targets (Fig. 13A). FEF inactivation did not significantly increase the rates of failing to make ipsiversive saccades (Fig. 13B).

\section{Results using random fixation locations}

In other step task experiments, we randomly varied the fixation location and used only flashed (30 ms duration) or persistent (1000 ms duration) targets. An example of an experiment using only persistent targets is shown in Fig. 14. Targets were spaced by $5 \mathrm{deg}$ to finely test the 
Fig. 14 Saccades made in a step task experiment that used only persistent targets. Conventions as in Fig. 3. Initial fixation location was central. Saccades made in response to contralateral and vertical targets before, during, and after FEF inactivation are shown

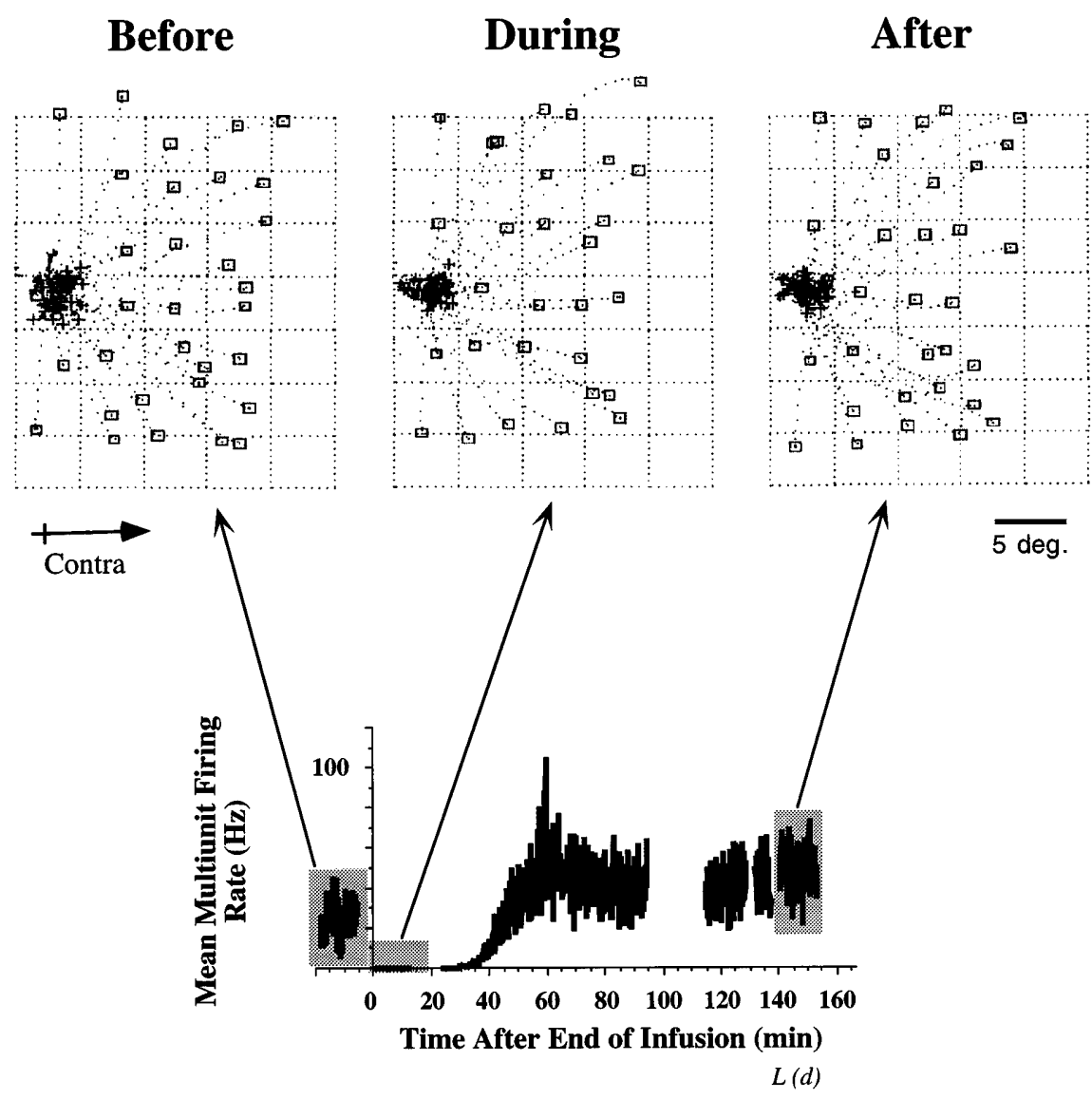

contralateral space. The one-third of trials that used central fixation are shown. Saccades made to ipsilateral targets are omitted to permit larger, more detailed illustration of the contraversive saccades. Even though FEF activity was thoroughly quenched (Fig. 14, bottom), the accuracy of contraversive saccades made to the persistent targets was only slightly perturbed (Fig. 14, During). Subtle upwards shifts in some saccades caused a small but significant increase in overall error (Fig. 15D, left). Similar effects were seen in the other experiment that used only persistent targets (Fig. 15C, left). In the two experiments using only flashed targets, contraversive saccades underwent the usual gamut of severe accuracy disruptions during FEF inactivation (similar to the impairments shown for 10 and $100 \mathrm{~ms}$ duration targets in Fig. 9; quantitative data shown in Fig. 15A,B, left). For all four experiments, the latencies of saccades made in response to contralateral targets were significantly increased during FEF inactivation (Fig. 15A-D, right). In one case the latency of saccades made to flashed ipsilateral targets was increased as well (Fig. 15A, right). In two saline experiments using only flashed targets, there were no effects (not shown).

Varying the initial fixation location had similar effects on saccades to flashed targets as was seen with saccades made in the delay task (Fig. 8). Ipsilateral fixation allowed for the generation of many contraversive but hypometric, correctly directed saccades during FEF inactivation (similar to effects shown in Fig. 8A, top panels).
Monkeys were impaired at foveating contralateral fixation LEDs during FEF inactivation, but in trials that were initiated successfully, the resulting ipsiversive saccades to flashed targets were normal and entered all parts of space (similar to data in Fig. 8B, top panels). In the two persistent target experiments, the only notable deficit was the problem of foveating contralateral fixation LEDs.

The only compelling evidence we obtained for an effect of FEF inactivation on saccadic dynamics came from the results of the step task experiments of Fig. 15. Saccades made to persistent contralateral targets exhibited a clear decrease in peak velocity over a large amplitude range from both central fixation (Fig. 16A) and ipsilateral fixation (Fig. 16B). Contraversive saccades made in response to flashed targets from ipsilateral fixation exhibited a distinct drop in peak velocity for saccades in the 5-15 deg range (Fig. 16C).

\section{Latency-error correlation}

In most of the delay and step task experiments, when saccades were made in response to contralateral targets during FEF inactivation, the saccades with greater errors also tended to have longer latencies (Fig. 17). This correlation was significant for six of the eight experimental conditions $(P<0.05$, regression lines are shown for the correlated data). 


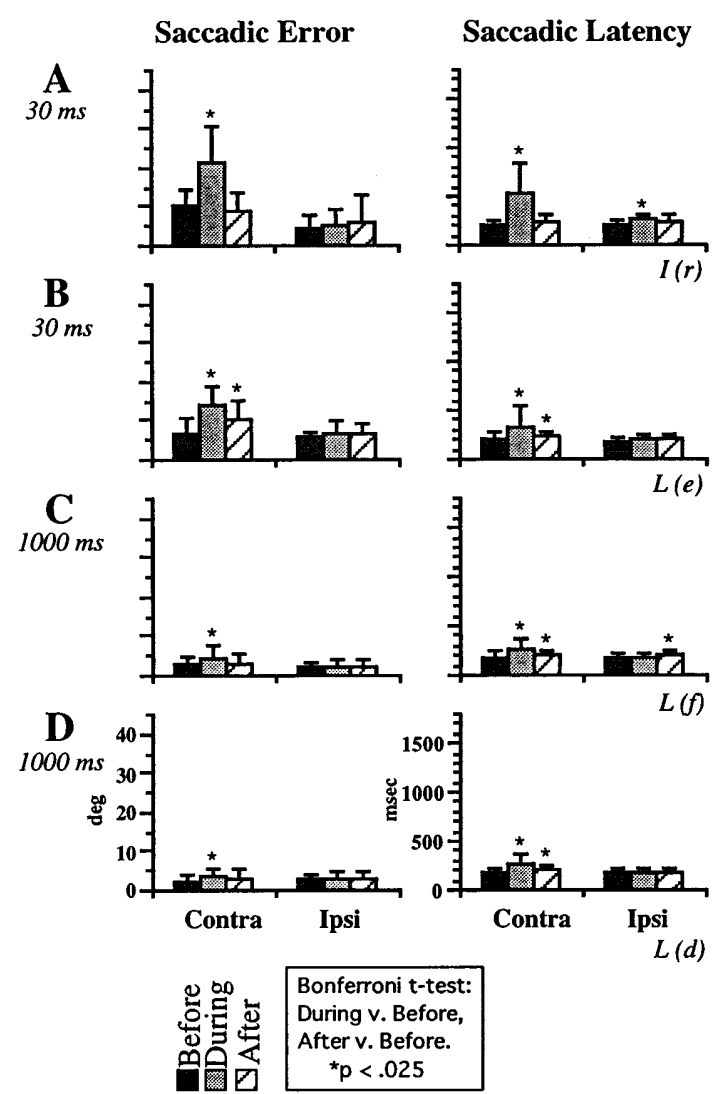

Fig. 15A-D Quantification of saccadic error and latency for the step task experiments that used only flashed or only persistent targets. All are lidocaine experiments. A,B Results of the two experiments using flashed targets. C,D Results of the two experiments using persistent targets. Degrees of freedom ranged from 25 to 108

\section{Fixation task}

The delay and step tasks did not rigorously test a monkey's ability to fixate. After a saccade in these tasks, only a brief fixation in the target window was required. Fixation deficits during FEF inactivation were suggested, however, by the impaired ability to foveate initial fixation LEDs located 20 deg contralaterally (e.g., Fig. 8B, During). To better examine this deficit, we ran monkeys on a formal fixation task. In two of three lidocaine infusions that used the fixation task, the monkey was severely impaired at fixating LEDs in contralateral space. A saline infusion to match the lidocaine volume caused no deficit.

Figure 18 shows the results of a fixation task experiment. Before left FEF inactivation (Fig. 18, Before), the monkey's initial fixation location in the dark was slightly up and ipsilateral. When an LED was lit at any of nine locations, the monkey made a saccade to it and then usually fixated near it for $5 \mathrm{~s}$. During FEF inactivation, the monkey's initial fixation location shifted ipsiversively (Fig. 18, During). The monkey made saccades toward the persistent contralateral LEDs but could not fixate
A

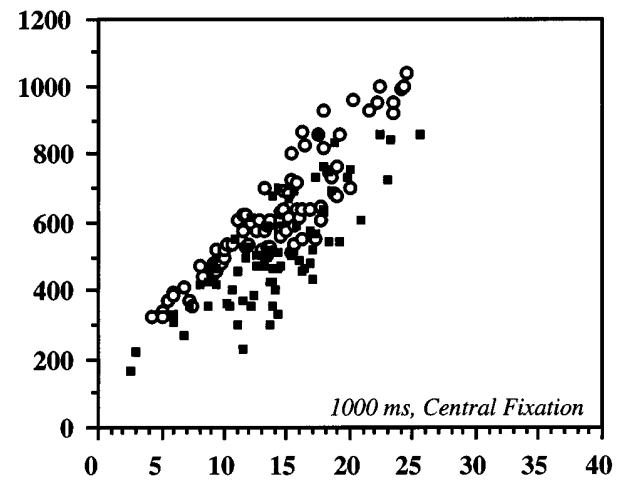

B
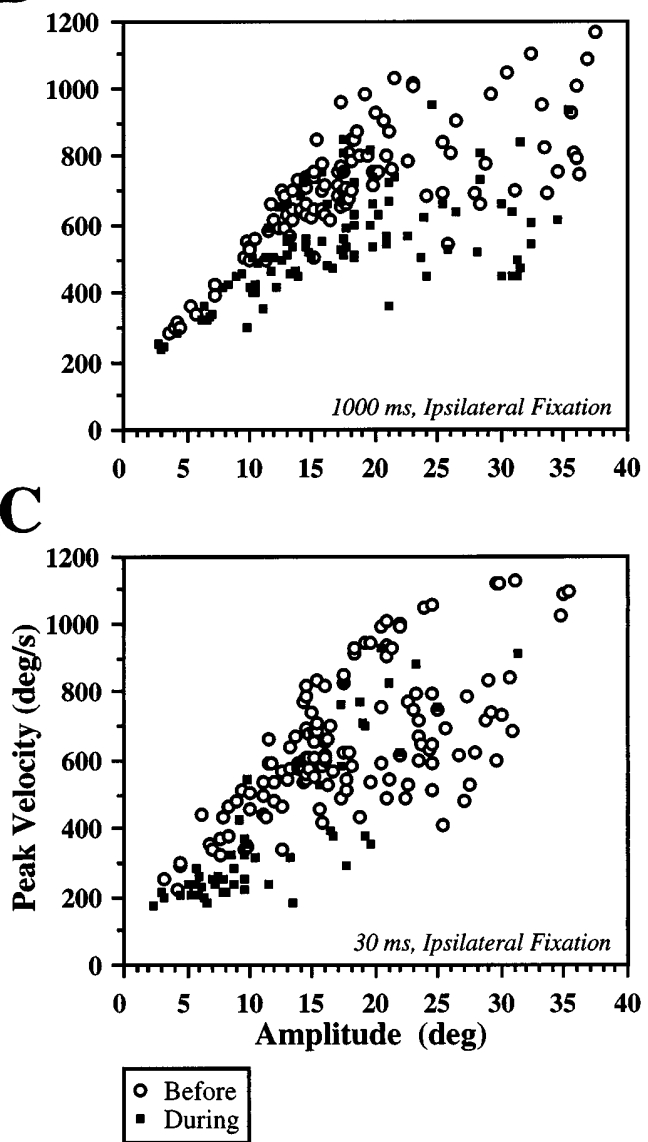

Fig. 16A-C Main sequences of saccades made in the step task experiments that used only flashed or only persistent targets. Data from the two flashed target experiments are pooled, as are the data from the two persistent target experiments. A Main sequences of contraversive saccades made to persistent contralateral targets from initially central fixation: saccades made before or during FEF inactivation are compared. B Same as A except that data are from the trials using initially ipsilateral fixation. $\mathbf{C}$ Main sequences of contraversive saccades made to flashed targets from initially ipsilateral fixation: saccades made before or during FEF inactivation are compared 
Fig. 17A-H Latency-error correlations of saccades made in response to contralateral targets during FEF inactivation.

Each datum is a saccade's error plotted against its latency.

Pooled results from each class

of experiment are depicted. All

data are from trials using ini-

tially central fixation. Regres-

sion lines are drawn in graphs

for which the data are correlated $(P<0.05)$. A Data from the four delay task experiments.

B-F Data from the step task experiments in which target duration was randomized. G Data from the step task experiments that used only flashed targets.

H Data from the step task experiments that used only persistent targets
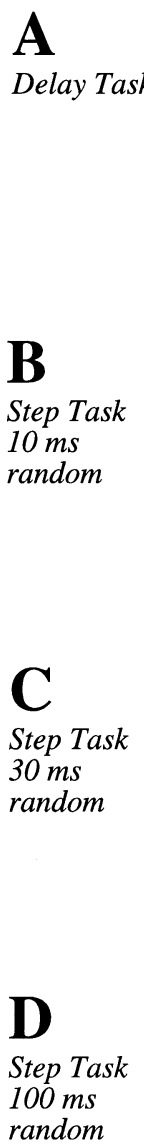

them steadily: the eyes drifted ipsiversively and upward at $1-5 \mathrm{deg} / \mathrm{s}$. Fixations of LEDs in ipsilateral and central space were less affected. After the inactivation (Fig. 18, After), the behavior recovered.

We did not do a muscimol infusion using the fixation task. Monkeys usually worked for less than $2 \mathrm{~h}$ at this task, presumably due to the long fixations required. We considered this to be too brief for an informative muscimol experiment. However, in a muscimol infusion that used various initial eye positions (experiment of Fig. 5D), the monkey was impaired at foveating the contralateral fixation LED, exhibiting deficits similar to those of Figure 18.

\section{Shift of eye position in the dark}

The ipsiversive shift in the eye's resting position in darkness during FEF inactivation was seen not only using the fixation task (ellipses, Fig. 18), but also during the intertrial intervals of the delay and step tasks. In every lido-

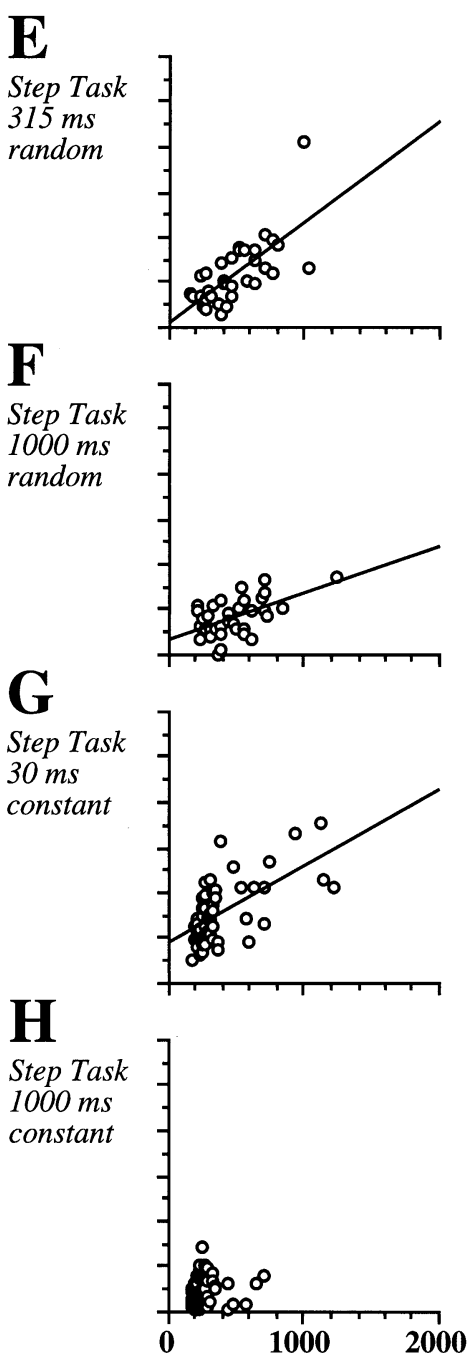

caine infusion, just after FEF inactivation there was a sharp ipsiversive shift in the mean fixation location in darkness (Fig. 19A). The average shift was highly significant (mean $-5.78 \mathrm{deg}$, SD $1.94 \mathrm{deg} ; t(11)=10.33$, $P<0.001)$. In saline infusions, shifts were not significant (Fig. 19C; mean $-0.09 \mathrm{deg}$, SD $3.07 \mathrm{deg} ; t(3)=0.06$, $P=0.956$ ). An ipsiversive shift was seen in both muscimol infusions, but it developed over a longer time-course (not shown). There was no ipsiversive shift seen in the saline infusion that matched the muscimol volume (not shown).

The vertical eye position also shifted just after lidocaine infusion (Fig. 19B), moving downward on average (mean shift $-1.72 \mathrm{deg}, \mathrm{SD} 1.95 \mathrm{deg} ; t(11)=3.052$, $P<0.05)$. The vertical eye position was unchanged just after saline infusion (Fig. 19D; mean shift $-0.78 \mathrm{deg}$, SD $1.37 \mathrm{deg} ; t(3)=1.134, P=0.339)$, but a downward shift tended to appear later. Gradually developing downward shifts also were seen in muscimol infusions and in the saline infusion used to match the muscimol volume (not shown). 


\section{Before}

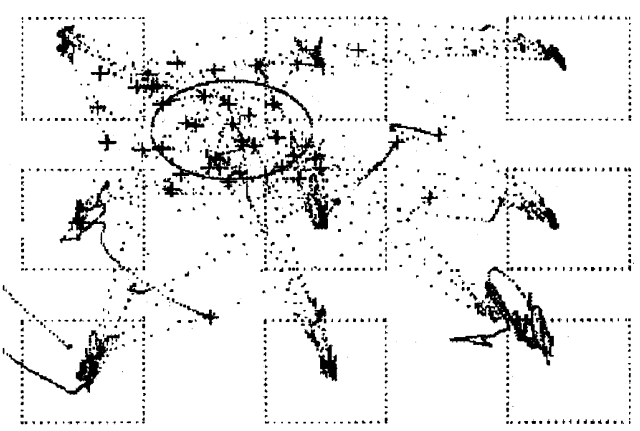

During
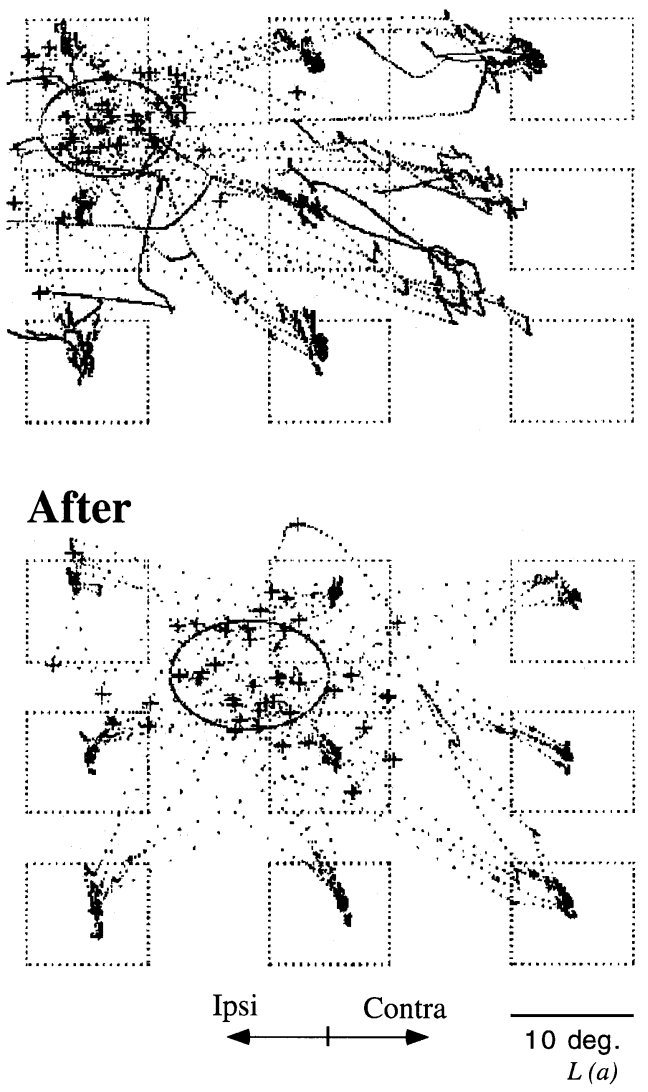

Fig. 18 Deficits in the foveation of contralateral LEDs using the fixation task. Initial eye position (crosses) and all the eye movements made during every trial are shown. Data from before, during, and after FEF inactivation are shown. Large dotted squares show the fixation windows around each LED. Center of the ellipse drawn on each illustration represents the mean initial fixation location of the eye, and the sizes of the horizontal and vertical axes of the ellipse are one standard deviation of the initial fixation location of the eye in each direction

\section{Discussion}

The fundamental result was that reversible inactivation of a portion of the FEF perturbed saccades and fixations. Monkeys were severely impaired at making contraversive saccades to locations of extinguished or flashed tar- gets, at fixating in contralateral space, and at suppressing ipsiversive saccades.

\section{Fundamental result}

Just after lidocaine infusion into the FEF, nearby unit activity was quenched. Severe saccadic impairments occurred in some tasks and fixational deficits usually appeared. These effects were followed by behavioral and neural recovery within the testing session. Muscimol infusion caused similar deficits, demonstrating that they were attributable to inactivation of cell bodies. Saline infusion caused negligible effects, showing that complications from pressure or dilution of extracellular space were minimal. The conclusion is that reversible inactivation of FEF neurons caused strong oculomotor perturbations. This implies that FEF neurons play necessary roles in generating saccades, in some task situations, and in maintaining fixation.

\section{Specific effects of FEF inactivation}

\section{Spatial aspects of saccades}

During FEF inactivation, monkeys made highly inaccurate saccades when attempting to look at locations of contralateral extinguished targets, in the delay task, or flashed targets, in the step task. Some saccades were contraversive but inaccurate and others were ipsiversive. Misdirected saccades were inaccurate from their moment of initiation: they went straight to the wrong place. This suggests that there was an impairment in encoding saccadic metrics. Ablations of FEF also cause deficits in making saccades to extinguished or flashed targets. Schiller and Sandell (1983) reported that FEF ablation caused a deficit in making saccades to brief targets (50-60 ms duration) in a step task, and Deng et al. (1986) found that FEF-lesioned monkeys were impaired at learning to make saccades to locations of extinguished targets in a delay task.

FEF inactivation caused only mild accuracy impairments for saccades made to persistent contralateral targets. This, too, agrees with the results of FEF ablation studies (Schiller et al. 1980; Deng et al. 1986; Lynch 1992). Why does silencing the FEF leave intact the ability to make reasonably accurate saccades to persistent targets even though it profoundly disrupts the accuracy of saccades made to extinguished or flashed targets?

The generation of any saccade requires involvement of brainstem circuitry (reviewed by Hepp et al. 1989). The FEF influences the brainstem saccade generator directly (Schiller et al. 1980; Segraves 1992), indirectly through the superior colliculus (SC) (Segraves and Goldberg 1987), and probably indirectly via nigral control of the SC (Hikosaka and Wurtz 1983, 1985; Stanton et al. 1988).

Models of the brainstem circuitry posit that it receives an input, which we will call $E_{d}$ (Fig. 20), which repre- 
Fig. 19A-D Mean resting eye position in darkness as a function of time after end of infusion. Just prior to a trial in every experiment, a fixation location of the eye was sampled.

Mean and standard deviations of these fixation locations were calculated for each block of data (e.g., see ellipses in Fig. 18). A Mean horizontal location of fixation in the dark for all 12 lidocaine infusions as a function of time. B Mean vertical location of fixation in the dark for the lidocaine infusions. $\mathbf{C}$

Mean horizontal location of fixation in the dark for four saline infusions used to match the lidocaine volume, as a function of time. D Mean vertical location of fixation in the dark for the saline infusions
A

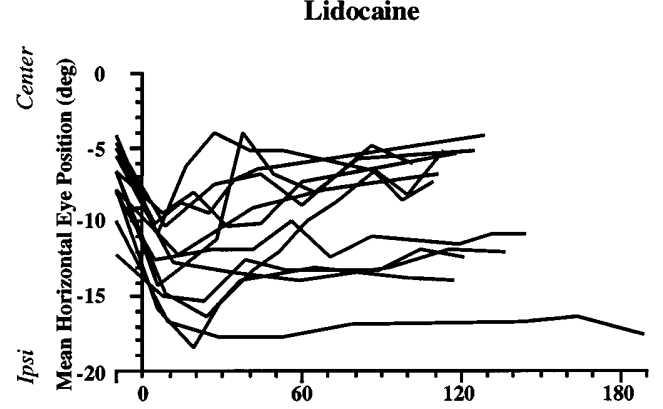

B

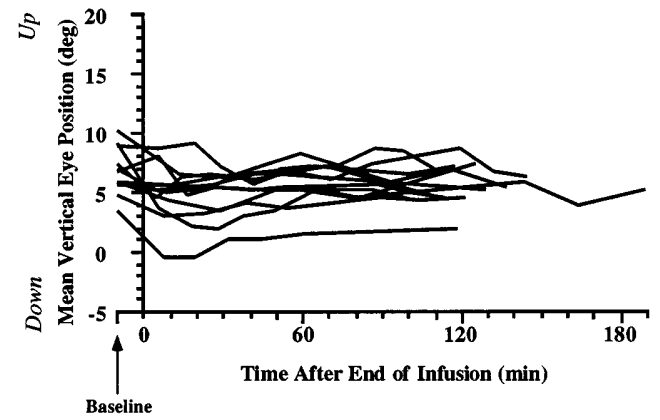

C

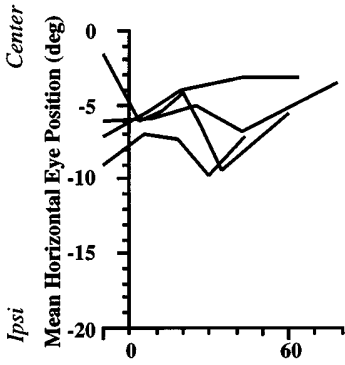

D

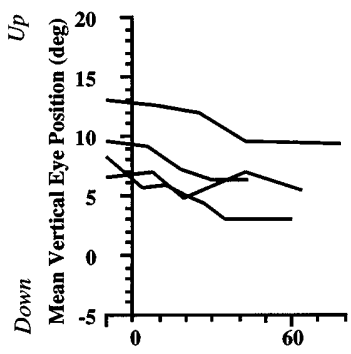

sents where the eye should go (Robinson 1975; van Gisbergen et al. 1981; Jürgens et al. 1981; Scudder 1988; van Gisbergen and van Opstal 1989; Waitzman et al. 1991). $E_{d}$ needs to be a sustained signal: it not only represents where to go but also drives the saccade. During a saccade, $E_{d}$ is compared with an estimate of how far the eye has moved. When the estimate equals the $E_{d}$ signal, the saccade terminates, and only then may $\mathrm{E}_{\mathrm{d}}$ cease. If a visual target is present throughout the saccade, visual responses of neurons will be available for generating $E_{d}$. However, if a target disappears before saccade initiation, the brain will have to maintain a representation of the target and make this representation available to the brainstem for generating $\mathrm{E}_{\mathrm{d}}$.

Some FEF presaccadic cells fire with tonic activity that is maintained long after a visual stimulus disappears, until a saccade is made (Bruce and Goldberg 1985; Funahashi et al. 1989). This leads to two hypotheses. The FEF may be a part of a cortical system for maintaining a neural representation of a target after it disappears. Therefore, inactivating the FEF might impair the ability to represent an absent target. Alternatively, since the FEF is an important mediator of saccadic signals from cortex to the brainstem (as discussed above), FEF inactivation might disrupt the brainstem's access to the neural representation of an absent target. Either way, FEF inactivation would cause $E_{d}$ to be disrupted in the absence of a target, resulting in the severe deficits of saccadic accuracy that we observed.

Monkeys made reasonably accurate saccades to persistent contralateral targets during FEF inactivation. These saccades may have been driven by an $\mathrm{E}_{\mathrm{d}}$ signal derived from direct visual responses, possibly provided to the brainstem through the occipitotectal pathway (Schiller 1977).

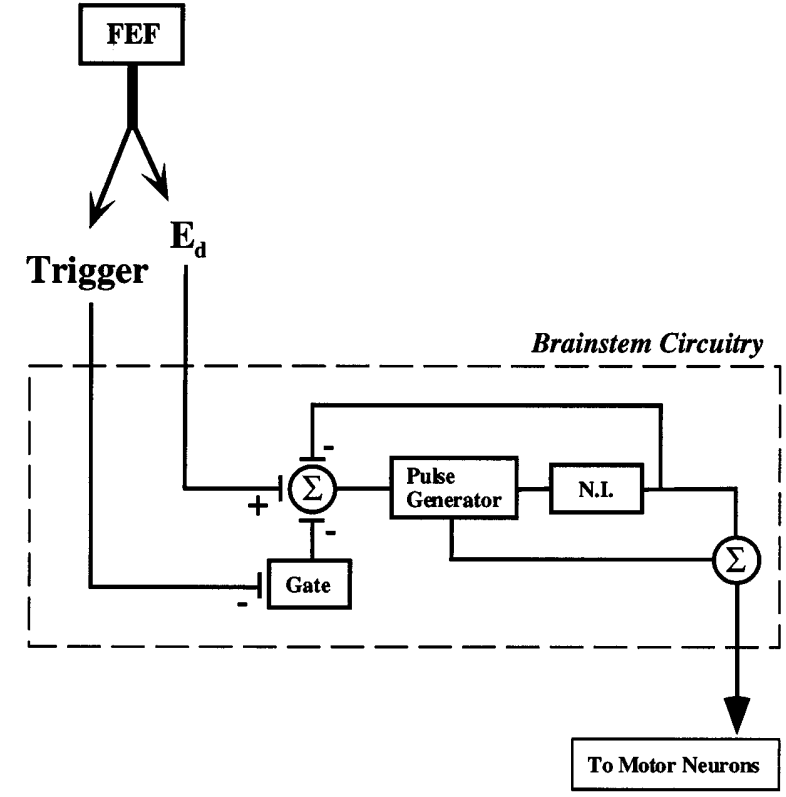

Fig. 20 Simplified model of brainstem circuitry subserving the generation of saccades. The inputs to the brainstem circuitry are $E_{\mathrm{d}}$ and a Trigger signal. The Trigger signal inhibits a Gate mechanism, permitting saccade initiation. The $\mathrm{E}_{\mathrm{d}}$ signal drives a Pulse Generator mechanism. A neural integrator (N.I.) generates an estimate of how far the eye has moved. This estimate is sent as negative feedback to cut off the $\mathrm{E}_{\mathrm{d}}$ input to the Pulse Generator. Our results and previous findings suggest that the FEF sends a common motor command downstream. The $\mathrm{E}_{\mathrm{d}}$ and Trigger signals are derived, at least in part, from this command

It is unlikely that the deficit in making saccades to locations of extinguished or flashed targets was due to an impairment at detecting the presentations of targets. FEF-inactivated monkeys demonstrated the ability to see the targets, since they made saccades toward them from 
ipsilateral fixation (e.g. Fig. 8A). It follows that they also saw the targets from central fixation, since visual detection abilities are fixed in reference to the retina and should not vary with eye position.

Dias et al. (1995) have independently confirmed the basic findings that we presented here. The major difference between our results is that, in their experiments that were similar to ours (their multiple target experiments), they found that saccades made in response to persistent contralateral targets were severely inaccurate during FEF inactivation. This difference in results might be related to differences in visual stimuli. Their targets, laser spots presented in dim ambient light, were less bright and were at lower contrast than our LEDs lit in darkness (E. C. Dias, personal communication). A short-term effect of FEF ablation is an elevation of the threshold for brightness perception (Latto 1977). A similar effect might occur when the FEF is temporarily inactivated. It may be that the persistent targets of Dias et al. (1995) were near or below the detection threshold whereas ours were suprathreshold. Note that when Dias et al. (1995) used persistent targets that were located more predictably, at only two possible locations, one of their monkeys did make accurate contraversive saccades to the targets during FEF inactivation. In general, we and they seem to agree that it is possible for FEF-inactivated monkeys to make reasonably accurate saccades to persistent contralateral targets.

We found that FEF-inactivated monkeys were less impaired at making saccades toward extinguished or flashed targets if initial fixation was ipsilateral rather than central. That is, contraversive centripetal saccades were less impaired than contraversive centrifugal ones. We think the simplest explanation for this is that from ipsilateral fixation, weakened contraversive saccadic attempts were aided by the natural centering tendencies of the eye musculature. Also, targets were more predictably located when fixation was ipsilateral, appearing only vertically and contralaterally. This increased predictability might have contributed to improved saccadic performance (Dias et al. 1995).

If a monkey initially fixated a contralateral LED during FEF inactivation, all ipsiversive saccades toward extinguished or flashed targets were accurate. Specifically, the saccades readily entered head-centered contralateral space, even though saccades seldom were made into this space from central or ipsilateral fixation. Therefore the deficit is not one of making saccades into contralateral space; rather, it is an impairment at making saccades in a contraversive direction. This implies that the intact FEF uses a "vector" code, signalling for saccades to be made contraversively, not a "place" code, signalling for saccades to be made to particular locations.

\section{Temporal aspects of saccades}

Besides an $\mathrm{E}_{\mathrm{d}}$ signal, the brainstem requires a trigger input to initiate a saccade (Fig. 20). The FEF seems to contribute to this trigger signal. During FEF inactivation, contraversive saccades made in all tasks had increased latency, implying that the trigger signal was generally delayed. This is consistent with the finding that FEF cell activity is related to the time of saccadic initiation (Segraves and Park 1993; Hanes et al. 1995). The FEF seems to make a particularly strong contribution to the triggering of saccades to locations of extinguished and flashed targets: saccades often were not initiated at all when these targets were used during FEF inactivation (Figs. 7B, 13).

We found inconsistent evidence that FEF inactivation influenced saccadic peak velocity. Saccades that should have been most affected, those made directly contraversively during FEF inactivation, sometimes had obviously decreased peak velocities (Fig. 9, " $\wedge$ " saccades; Fig. 16) but sometimes they did not (Figs. 6D, 12C). Saccades made to persistent targets seemed as likely to have their velocity lowered as saccades made to flashed targets (e.g., Fig. 16). Our mixed results do not help to resolve the controversy over whether the FEF is involved in controlling saccadic velocity. Schiller et al. (1979b, 1980) found that FEF ablation lowered saccadic velocity when monkeys were required to scan a board of apple pieces, and Deng et al. (1986) reported that FEF ablation lowered the velocity of saccades made to locations of extinguished targets. However, in a recording study, Segraves and Park (1993) found little evidence for FEF involvement in the control of dynamical aspects of saccades, whether the saccades were made to flashed or persistent targets in a step task or to extinguished targets in a delay task. The role of the FEF in controlling saccadic velocity remains unclear.

\section{Latency-error correlation}

A general effect of FEF inactivation was that the latencies and errors of saccades made in response to contralateral targets usually were directly correlated. Although trial-by-trial variations in error and latency during FEF inactivation were to be expected because of probable slight variations in the strength of the deficit over contralateral space and over time, the fact that error and latency covaried was surprising.

The probable basis for this correlation is that information about where a saccade should go and when it should begin is encoded in a common motor command leaving the FEF (Fig. 20). It is known that individual FEF movement neurons signal both where and when to make a saccade. "Where to go" is represented by a cell's location in the FEF (Robinson and Fuchs 1969; Bruce and Goldberg 1985; Bruce et al. 1985) and "when to go" is represented in a cell's firing rate (Segraves and Park 1993; Hanes et al. 1995). As noted above, these FEF signals influence the brainstem directly and indirectly. We therefore suggest the following scenario to explain the latency-error correlation. When a portion of the FEF is inactivated, the integrity of a saccadic command originating from the rest of the structure is disrupted, varying from trial to tri- 
al. Because brainstem signals that determine where and when to make a saccade $\left(E_{d}\right.$ and trigger signals, respectively) are at least partly derived from this disrupted common motor command, there is a tendency for saccadic accuracy and latency to covary from trial to trial.

\section{Premature ipsiversive saccades}

We found that FEF inactivation increased the rate of making premature saccades to ipsilateral targets in the delay task (also found by Dias et al. 1995). This implies that a role of the FEF is to suppress inappropriate ipsiversive saccades. Previous results using other methods had suggested that the FEF suppresses saccades, but selectivity for ipsiversive saccades had not been found. Two studies reported that electrical stimulation in the FEF can suppress saccades, but one found that both contraversive and ipsiversive saccades were affected (Burman and Bruce 1990) and the other did not describe the directionality of the suppression (Dassonville et al. 1992). Guitton et al. (1985) found that unilateral frontal lobe lesions in humans cause deficits in withholding saccades to visual stimuli, but the effects were bilateral.

\section{Fixation}

In the fixation task, FEF-inactivated monkeys often were severely impaired at foveating contralateral LEDs for extended periods. A saccade was made toward the persistent target but fixation was not maintained at the new position. Rather, the eye was swept ipsiversively and upward (Fig. 18, During). Two factors may have contributed to this phenomenon. First, fixation itself seemed to be weakened since the eye did not "hold its ground" after entering contralateral space. Second, there may have been a neural imbalance at the brainstem that caused the ipsiversive component of the drifting, since signals from the FEF in the other hemisphere presumably were not weakened.

The results of the fixation task, along with observations that FEF-inactivated monkeys were impaired at fixating contralateral fixation LEDs during the delay and step tasks, are consistent with a hypothesis that the FEF is involved with maintaining fixation. This concurs with results of previous studies demonstrating that fixation signals are carried by some FEF neurons (Bizzi 1968; Bizzi and Schiller 1970; Suzuki and Azuma 1977; Bruce and Goldberg 1985), many of which project to subcortical oculomotor regions (Segraves and Goldberg 1987; Segraves 1992). Our results also are in agreement with the findings of Latto and Cowey (1971b) that FEF lesions cause deficits in fixating contralaterally.

\section{Ipsiversive shift of eye position in darkness}

FEF inactivation always caused an ipsiversive shift of eye position in the dark. As discussed above, during FEF inactivation, the ability to fixate is poor in contralateral space, the eyes drift ipsiversively, and generation of contraversive saccades is impaired in the absence of persistent visual stimulation. The combination of these effects probably caused the eye position to shift ipsiversively in darkness. We also found a slight downward shift, an effect we cannot explain. Ablation of FEF also causes ipsiversive and downward shifts in eye position (Latto and Cowey 1971b).

\section{Comparison of FEF inactivation with SC inactivation}

The FEF and the SC both play important roles in generating saccades to visual stimuli (Schiller et al. 1980). We will compare our FEF inactivation results with previously reported results of SC inactivation, with the caveat that such comparison is indirect since no investigator has reversibly inactivated both the FEF and SC using identical methods.

There are many similarities between FEF inactivation and SC inactivation. During inactivation of either structure, the accuracies of saccades made in attempt to reach locations of extinguished targets are more disrupted than the accuracies of saccades made to persistent targets (Hikosaka and Wurtz 1985). Other similarities between FEF and SC inactivation include latency increases for saccades made in delay tasks (Hikosaka and Wurtz 1985) and step tasks (Hikosaka and Wurtz 1985; Schiller et al. 1987), impaired fixation (Hikosaka and Wurtz 1985; Schiller et al. 1987; Munoz and Wurtz 1993), and an ipsiversive shift in the resting position of the eyes (Hikosaka and Wurtz 1985; Schiller et al. 1987).

There are three major differences between FEF and $\mathrm{SC}$ inactivation. The first concerns saccades made to persistent targets. During SC inactivation, contraversive saccades made in response to persistent targets are consistently inaccurate (Hikosaka and Wurtz 1985, 1986; Lee et al. 1988) to a degree that clearly exceeds the impairment seen by us during FEF inactivation. Also, during some SC inactivations, contraversive saccades to persistent targets are triggered only infrequently, and sometimes they are not triggered at all (Hikosaka and Wurtz 1985). In contrast, we never saw significant rates of failing to trigger saccades to persistent targets in our FEF inactivations. We are led to conclude that the FEF is less important than the SC for generating saccades to persistent targets.

The second difference concerns ipsiversive premature saccades. Inactivation of the SC has not been reported to cause the appearance of premature saccades in a delay task (Hikosaka and Wurtz 1985) unless the inactivation is aimed at the rostral SC (Munoz and Wurtz 1993). Premature saccades caused by rostral SC inactivation have very short latencies $(\sim 100 \mathrm{~ms})$ and are predominantly contraversive (Munoz and Wurtz 1993). In contrast, FEF inactivation results in premature saccades that are of long latency $(\geq 195 \mathrm{~ms})$ and are ipsiversive. Since inactivation of homolateral FEF or rostral SC causes such dif- 
ferent kinds of premature saccades, but FEF projects much more to the ipsilateral than the contralateral SC (Distel and Fries 1982), it is illogical that FEF inactivation causes premature saccades through a silencing of efferents to rostral SC. A simpler explanation is that, through transcallosal projections (Huerta et al. 1987), one FEF normally inhibits the contralateral one (Schlag et al. 1996). Inactivation of an FEF therefore would disinhibit the contralateral FEF and promote the premature triggering of ipsiversive saccades.

The third major difference in the effects of FEF and $\mathrm{SC}$ inactivation concerns saccadic velocity. Silencing the SC with lesions (Schiller et al. 1980) or reversible inactivation (Hikosaka and Wurtz 1985, 1986; Lee et al. 1988; Munoz and Wurtz 1993) causes reliable, pronounced velocity deficits. FEF lesions also cause velocity deficits, as discussed above, but reversible FEF inactivation causes ambiguous effects: we found inconsistent, mild velocity deficits, but Dias et al. (1995, and personal communication) found reliable deficits. A hypothesis consistent with all these findings is that the $\mathrm{SC}$, in comparison with the $\mathrm{FEF}$, is more intimately associated with structures that control saccadic velocity (see also the Discussions of Hikosaka and Wurtz 1985, and Segraves and Park 1993).

\section{Conclusion}

This study revealed at least three possible roles of the FEF in natural behavior. First, we found that the FEF is necessary for generating contraversive saccades to locations of targets that have disappeared. The finding that the FEF is needed to make saccades to extinguished targets in the delay task, although informative, does not have obvious relevance to everyday behavior. It is unclear why, in natural situations, a monkey would purposefully withhold a saccade toward a target until after it disappears. More suggestive is the result from using flashed targets in the step task: the FEF is needed for the natural behavior of making a contraversive saccade to something seen fleetingly, "out of the corner of the eye."

Second, we found that the FEF contributes to fixating in contralateral space. This complements the saccadic role of this structure, since a typical outcome of making a contraversive saccade is to put the eyes in a contralateral position.

Finally, we want to emphasize that the use of reversible inactivation seems to have revealed a major function of the FEF that was missed by the use of other techniques. The FEF is needed for suppressing inappropriate ipsiversive saccades as well as for generating appropriate contraversive saccades.

Acknowledgements The support and advice of Peter Schiller are gratefully acknowledged. We thank Elisa Dias for many interesting and constructive communications regarding our results. We also thank Robert Wurtz, Michael Goldberg, Christian Quaia, Martin Paré, and the two anonymous referees for helpful ideas and comments. The drawing of the brain in Fig. 1 is from a photograph taken by Peter Schiller and Karl Zipser. This work was supported by NEI EY-08502 to Peter Schiller.

\section{References}

Bizzi E (1968) Discharge of frontal eye field neurons during saccadic and following eye movements in unanesthetized monkeys. Exp Brain Res 6:69-80

Bizzi E, Schiller PH (1970) Single unit activity in the frontal eye fields of unanesthetized monkeys during eye and head movement. Exp Brain Res 10:151-158

Bruce CJ, Goldberg ME (1985) Primate frontal eye fields. I. Single neurons discharging before saccades. J Neurophysiol 53:603-635

Bruce CJ, Goldberg ME, Bushnell MC, Stanton GB (1985) Primate frontal eye fields. II. Physiological and anatomical correlates of electrically evoked eye movements. J Neurophysiol 54:714-734

Burman D, Bruce CJ (1990) Suppression and delay of saccades by microstimulation in the macaque frontal eye fields. Invest Ophthalmol Vis Sci [Suppl] 31:400

Dassonville P, Schlag J, Schlag-Rey M (1992) The frontal eye field provides the goal of saccadic eye movement. Exp Brain Res 89:300-310

Deng S-Y, Goldberg ME, Segraves MA, Ungerleider LG, Mishkin M (1986) The effect of unilateral ablation of the frontal eye fields on saccadic performance in the monkey. In: Keller E, Zee DS (eds) Adaptive processes in the visual and oculomotor systems. Pergamon Press, Oxford, pp 201-208

Dias EC, Kiesau M, Segraves MA (1995) Acute activation and inactivation of macaque frontal eye field with GABA-related drugs. J Neurophysiol 74:2744-2748

Distel H, Fries W (1982) Contralateral cortical projections to the superior colliculus in the macaque monkey. Exp Brain Res 48:157-162

Ferrier D (1875) Experiments on the brains of monkeys. Proc R Soc Lond 23:409-430

Funahashi S, Bruce CJ, Goldman-Rakic PS (1989) Mnemonic coding of visual space in the monkey's dorsolateral prefrontal cortex. J Neurophysiol 61:331-349

Gnadt JW, Bracewell RM, Andersen RA (1991) Sensorimotor transformation during eye movements to remembered visual targets. Vision Res 31:693-715

Goldberg ME, Bruce CJ (1990) Primate frontal eye fields. III. Maintenance of a spatially accurate saccade signal. J Neurophysiol 64:489-508

Guitton D, Buchtel HA, Douglas RM (1985) Frontal lobe lesions in man cause difficulties in suppressing reflexive glances and in generating goal-directed saccades. Exp Brain Res 58:455-472

Hanes DP, Thompson KG, Schall JD (1995) Relationship of presaccadic activity in frontal eye field and supplementary eye field to saccade initiation in macaque: Poisson spike train analysis. Exp Brain Res 103:85-96

Hepp K, Henn V, Vilis T, Cohen B (1989) Brainstem regions related to saccade generation. In: Wurtz RH, Goldberg ME (eds) Reviews of oculomotor research, vol 3. The neurobiology of saccadic eye movements. Elsevier, Amsterdam, pp 101-212

Hikosaka O, Wurtz RH (1983) Visual and oculomotor functions of monkey substantia nigra pars reticulata. IV. Relation of substantia nigra to superior colliculus. J Neurophysiol 49:1285-1301

Hikosaka O, Wurtz RH (1985) Modification of saccadic eye movements by GABA-related substances. I. Effect of muscimol and bicuculline in monkey superior colliculus. J Neurophysiol 53:266-291

Hikosaka O, Wurtz RH (1986) Saccadic eye movements following injection of lidocaine into the superior colliculus. Exp Brain Res 61:531-539

Huerta MF, Krubitzer LA, Kaas JH (1987) Frontal eye field as defined by intracortical microstimulation in squirrel monkeys, owl monkeys, and macaque monkeys. II. Cortical connections. J Comp Neurol 265:332-361

Judge SJ, Richmond BJ, Chu FC (1980) Implantation of magnetic search coils for measurement of eye position: an improved method. Vision Res 20:535-538 
Jürgens R, Becker W, Kornhuber HH (1981) Natural and drug-induced variations of velocity and duration of human saccadic eye movements: evidence for a control of the neural pulse generator by local feedback. Biol Cybern 39:87-96

Kurata K, Hoffman DS (1994) Differential effects of muscimol microinjection into dorsal and ventral aspects of the premotor cortex of monkeys. J Neurophysiol 71:1151-1164

Latto R (1977) The effects of bilateral frontal eye-field, posterior parietal or superior collicular lesions on brightness thresholds in the rhesus monkey. Neuropsychologia 15:507-516

Latto R, Cowey A (1971a) Visual field defects after frontal eyefield lesions in monkeys. Brain Res 30:1-24

Latto R, Cowey A (1971b) Fixation changes after frontal eye-field lesions in monkeys. Brain Res 30:25-36

Lee C, Rohrer WH, Sparks DL (1988) Population coding of saccadic eye movements by neurons in the superior colliculus. Nature 332:357-360

Lynch JC (1992) Saccade initiation and latency deficits after combined lesions of the frontal and posterior eye fields in monkeys. J Neurophysiol 68:1913-1916

Munoz DP, Wurtz RH (1993) Fixation cells in the monkey superior colliculus. II. Reversible activation and deactivation. J Neurophysiol 70:576-589

Ohtsuka K, Sato H, Noda H (1994) Saccadic burst neurons in the fastigial nucleus are not involved in compensating for orbital nonlinearities. J Neurophysiol 71:1976-1980

Ragsdale DS, McPhee JC, Scheuer T, Catterall WA (1994) Molecular determinants of state-dependent block of $\mathrm{Na}^{+}$channels by local anesthetics. Science 265:1724-1728

Ritchie JM (1979) A pharmacological approach to the structure of sodium channels in myelinated axons. Ann Rev Neurosci 2:341-362

Robinson DA (1963) A method of measuring eye movement using a scleral search coil in a magnetic field. IEEE Trans in Biomed Electronics 10:137-145

Robinson DA (1975) Oculomotor control signals. In: Lennerstrand G, Back-y-Rita P (eds) Basic mechanisms of ocular motility and their clinical implications. Pergamon Press, Oxford, pp 337-374

Robinson DA, Fuchs AF (1969) Eye movements evoked by stimulation of the frontal eye fields. J Neurophysiol 32:637-648

Robinson FR, Straube A, Fuchs AF (1993) Role of caudal fastigial nucleus in saccade generation. II. Effects of muscimol inactivation. J Neurophysiol 70:1741-1758

Russo GS, Bruce CJ (1993) Effect of eye position within the orbit on electrically elicited saccadic eye movements: a comparison of the macaque monkey's frontal and supplementary eye fields. J Neurophysiol 69:800-818

Schall JD (1991) Neuronal activity related to visually guided saccades in the frontal eye fields of rhesus monkeys: comparison with supplementary eye fields. J Neurophysiol 66:559-579

Schiller PH (1977) The effect of superior colliculus ablation on saccades elicited by cortical stimulation. Brain Res 122:154-156
Schiller PH, Sandell JH (1983) Interactions between visually and electrically elicited saccades before and after superior colliculus and frontal eye field ablations in the rhesus monkey. Exp Brain Res 49:381-392

Schiller PH, True SD, Conway JL (1979a) Paired stimulation of the frontal eye fields and the superior colliculus of the rhesus monkey. Brain Res 179:162-164

Schiller PH, True SD, Conway JL (1979b) Effects of frontal eye field and superior colliculus ablations on eye movements. Science 206:590-592

Schiller PH, True SD, Conway JL (1980) Deficits in eye movements following frontal eye-field and superior colliculus ablations. J Neurophysiol 44:1175-1189

Schiller PH, Sandell JH, Maunsell JHR (1987) The effect of frontal eye field and superior colliculus lesions on saccadic latencies in the rhesus monkey. J Neurophysiol 57:1033-1049

Schlag J, Dassonville P, Schlag-Rey M, Letinsky MS (1996) Functional interactions between the two frontal eye fields in monkey. Soc Neurosci Abstr 22:1457

Scudder CA (1988) A new local feedback model of the saccadic burst generator. J Neurophysiol 59:1455-1475

Segraves MA (1992) Activity of monkey frontal eye field neurons projecting to oculomotor regions of the pons. J Neurophysiol 68:1967-1985

Segraves MA, Goldberg ME (1987) Functional properties of corticotectal neurons in the monkey's frontal eye field. J Neurophysiol 58:1387-1419

Segraves MA, Park K (1993) The relationship of monkey frontal eye field activity to saccade dynamics. J Neurophysiol 69:1880-1889

Sommer MA (1994) Express saccades elicited during visual scan in the monkey. Vision Res 34:2023-2038

Stanton GB, Goldberg ME, Bruce CJ (1988) Frontal eye field efferents in the macaque monkey. I. Subcortical pathways and topography of striatal and thalamic terminal fields. J Comp Neurol 271:473-492

Suzuki H, Azuma M (1977) Prefrontal neuronal activity during gazing at a light spot in the monkey. Brain Res 126:497-508

Tehovnik EJ, Sommer MA (1997) Effective spread and timecourse of neural inactivation caused by lidocaine injection in monkey cerebral cortex. J Neuroscie Methods 74:17-26

van Gisbergen JAM, van Opstal AJ (1989) Models. In: Wurtz RH, Goldberg ME (eds) Reviews of oculomotor research, vol 3. The neurobiology of saccadic eye movements. Elsevier, Amsterdam, pp 69-101

van Gisbergen JAM, Robinson DA, Gielen S (1981) A quantitative analysis of generation of saccadic eye movements by burst neurons. J Neurophysiol 45:417-442

Waitzman DM, Ma TP, Optican LM, Wurtz RH (1991) Superior colliculus neurons mediate the dynamic characteristics of saccades. J Neurophysiol 66:1716-1737

White JM, Sparks DL, Stanford TR (1994) Saccades to remembered target locations: an analysis of systematic and variable errors. Vision Res 34:79-92 